\title{
Two-step estimation of a multivariate Lévy process
}

\author{
Habib Esmaeili * Claudia Klüppelberg ${ }^{\dagger}$
}

June 8, 2018

\begin{abstract}
Based on the concept of a Lévy copula to describe the dependence structure of a multivariate Lévy process we present a new estimation procedure. We consider a parametric model for the marginal Lévy processes as well as for the Lévy copula and estimate the parameters by a two-step procedure. We first estimate the parameters of the marginal processes, and then estimate in a second step only the dependence structure parameter. For infinite Lévy measures we truncate the small jumps and base our statistical analysis on the large jumps of the model. Prominent example will be a bivariate stable Lévy process, which allows for analytic calculations and, hence, for a comparison of different methods. We prove asymptotic normality of the parameter estimates from the two-step procedure and, in particular, we derive the Godambe information matrix, whose inverse is the covariance matrix of the normal limit law. A simulation study investigates the loss of efficiency because of the two-step procedure and the truncation.
\end{abstract}

AMS 2000 Subject Classifications: 62F10, 62F12, 62M05.

Keywords: dependence structure, Godambe information matrix, IFM, inference functions for margins, Lévy copula, maximum likelihood estimation, multivariate Lévy process, reduced likelihood, two-step parameter estimation

\footnotetext{
${ }^{*}$ Center for Mathematical Sciences, Technische Universität München, Boltzmannstraße 3, 85748 Garching, Germany, email: esmaeili@ma.tum.de, http://www-m4.ma.tum.de/

${ }^{\dagger}$ Center for Mathematical Sciences, and Institute for Advanced Study, Technische Universität München, Boltzmannstraße 3, 85748 Garching, Germany, email: cklu@ma.tum.de, http://www-m4.ma.tum.de/
} 


\section{Introduction}

In Esmaeili and Klüppelberg [7] we presented the maximum likelihood estimation (MLE) for a bivariate stable subordinator. We assumed for the marginal subordinators to be both stable with the same parameters and modeled the dependence structure by a Clayton Lévy copula. Estimation was based on observed jumps larger than some predefined $\varepsilon>0$ in both components within a fixed interval $[0, t]$. For this model we computed the MLEs numerically and proved asymptotic normality for $\varepsilon \rightarrow 0$ and/or for $t \rightarrow \infty$, respectively. It is certainly useful to know that such a procedure works; but for more general models as, for instance, for higher dimensional models with different marginal Lévy processes, this estimation method becomes computationally very expensive.

Consequently, we present in this paper an alternative, which is a Lévy equivalent of the so-called IFM (inference functions for margins) method, a standard method in multivariate statistics; cf. Godambe [8], Joe [10], Ch. 10, and Xu [15], Ch. 2. The observation scheme as chosen in Esmaeili and Klüppelberg [7] was simple in the sense that we only considered observations with jumps in both components larger than some $\varepsilon>0$. For this observation scheme, however, the marginally truncated processes are not independent of the Lévy copula parameter.

The appropriate observation scheme to separate marginal and dependence parameters of the small jumps truncated processes requires to consider each component process separately and observe jumps larger than $\varepsilon$ in each single component. This results again in a compound Poisson process (CPP), where jumps larger than $\varepsilon$ in both components are seen as joint jumps, and those jumps with sizes larger than $\varepsilon$ only in one component (and smaller in the other) are treated as positive jumps in one component and jump size 0 in the other.

Separation of the marginals and the Lévy copula is based on Sklar's theorem for Lévy measures. Due to the fact that all Lévy processes with the exception of a CPP have a singularity

in 0 , the Lévy measure is considered on quadrants in $\mathbb{R}^{d}$ avoiding the origin. The simplest object to consider is, hence, a $d$-dimensional subordinator, which allows for only positive jumps in all components. We restrict ourselves in this paper to such processes, extensions to general Lévy processes are not difficult, but notationally involved; see Kallsen and Tankov [11] or Eder and Klüppelberg [4].

Our paper is organised as follows. In Section 2 we introduce the notion of a Lévy copula needed later to model the dependence structure between the components of a multivariate Lévy process. Here we also explain the truncation scheme of the observed jumps and present our prominent example, the bivariate $\alpha$-stable Clayton subordinator. Section 3 is dedicated to the two-step estimation procedure. We prove consistency and asymptotic normality of the IFM estimates in Section 4 including the calculation of the covariance matrix as the inverse of the Godambe information matrix. For a comparison with the MLE based on the full model we calculate its log-likelihood function in Section 5 . Finally, in Section 6, we perform a small simulation study, where we compare the quality of all three estimation methods: the full MLE, the full MLE based on joint jumps only, and the estimates from the two-step procedure. 


\section{Preliminaries}

\section{The Lévy copula}

Throughout this paper we denote by $\mathbf{S}=(\mathbf{S}(t))_{t \geq 0}$ an increasing Lévy process with values in $\mathbb{R}_{+}^{d}$ defined on a filtered probability space $\left(\Omega, \mathcal{F},\left(\mathcal{F}_{t}\right)_{t \geq 0}, \mathbb{P}\right)$. This means that $\mathbf{S}$ is a subordinator without Gaussian component, drift $\gamma$ and a Lévy measure $\Pi$ on $\mathbb{R}_{+}^{d}$ satisfying $\Pi(\{\mathbf{0}\})=0$ and $\int_{\mathbb{R}_{+}^{d}} \min \{x, 1\} \Pi(d x)<\infty$; cf. Sato [13], Th. 21.5, or Cont and Tankov [3], Prop. 3.10.

The tail integral of the Lévy measure $\Pi$ is the function $\bar{\Pi}:[0, \infty]^{d} \rightarrow[0, \infty]$ defined by

$$
\bar{\Pi}\left(x_{1}, \ldots, x_{d}\right)= \begin{cases}\Pi\left(\left[x_{1}, \infty\right) \times \cdots \times\left[x_{d}, \infty\right)\right), & \left(x_{1}, \ldots, x_{d}\right) \in[0, \infty)^{d} \backslash\{\mathbf{0}\} \\ 0, & x_{i}=\infty \text { for at least one } i \\ \infty, & \left(x_{1}, \ldots, x_{d}\right)=\mathbf{0} .\end{cases}
$$

The marginal tail integrals are defined analogously for $i=1, \ldots, d$ as $\bar{\Pi}_{i}(x)=\Pi_{i}([x, \infty))$ for $x \geq 0$; cf. Cont and Tankov [3, Def. 5.7, and Kallsen and Tankov [11, Def. 3.3 and 3.4.

The jump dependence of the process $\mathbf{S}$ is part of the multivariate tail integral and can be described by a so-called Lévy copula. We recall the notion of a Lévy copula from [3, 11] to be a measure defining function $\mathfrak{C}^{\mathfrak{1}}:[0, \infty]^{d} \rightarrow[0, \infty]$ with Lebesgue margins $\mathfrak{C}_{k}(u)=u$ for all $u \in[0, \infty]$ and $k=1, \ldots, d$.

The following result, called Sklar's Theorem for Lévy copulas, is central for our set-up; it has been proved in Cont and Tankov [3], Th. 5.4, for a bivariate Lévy process and in Kallsen and Tankov [11], Th. 3.6, for a $d$-dimensional Lévy process.

Theorem 2.1. Let $\bar{\Pi}$ denote the tail integral of a spectrally positive d-dimensional Lévy process, whose components have Lévy measures $\Pi_{1}, \ldots, \Pi_{d}$. Then there exists a Lévy copula $\mathfrak{C}:[0, \infty]^{d} \rightarrow$ $[0, \infty]$ such that for all $x_{1}, x_{2}, \ldots, x_{d} \in[0, \infty]$

$$
\bar{\Pi}\left(x_{1}, \ldots, x_{d}\right)=\mathfrak{C}\left(\bar{\Pi}_{1}\left(x_{1}\right), \ldots, \bar{\Pi}_{d}\left(x_{d}\right)\right) .
$$

If the marginal tail integrals are continuous, then this Lévy copula is unique. Otherwise, it is unique on $\operatorname{Ran}\left(\bar{\Pi}_{1}\right) \times \cdots \times \operatorname{Ran}\left(\bar{\Pi}_{d}\right)$.

Conversely, if $\mathfrak{C}$ is a Lévy copula and $\bar{\Pi}_{1}, \ldots, \bar{\Pi}_{d}$ are one-dimensional tail integrals of spectrally positive Lévy processes, then the relation (2.2) defines the tail integral of a d-dimensional spectrally positive Lévy process and $\bar{\Pi}_{1}, \ldots, \bar{\Pi}_{d}$ are tail integrals of its components.

\section{Truncation of the small jumps}

For notational convenience we proceed with a bivariate subordinator. As truncation point we choose $\varepsilon>0$. Figure 1 shows how the Lévy measure $\Pi$ on $\mathbb{R}_{+}^{2} \backslash(0, \varepsilon)^{2}$ is divided into two parts, the part concentrated on $[\varepsilon, \infty)^{2}$, and the part concentrated on the axes, which is in fact the projected measure of $\Pi$ on $[\varepsilon, \infty) \times(0, \varepsilon)$ and $(0, \varepsilon) \times[\varepsilon, \infty)$ to the horizontal and vertical axes, respectively. 

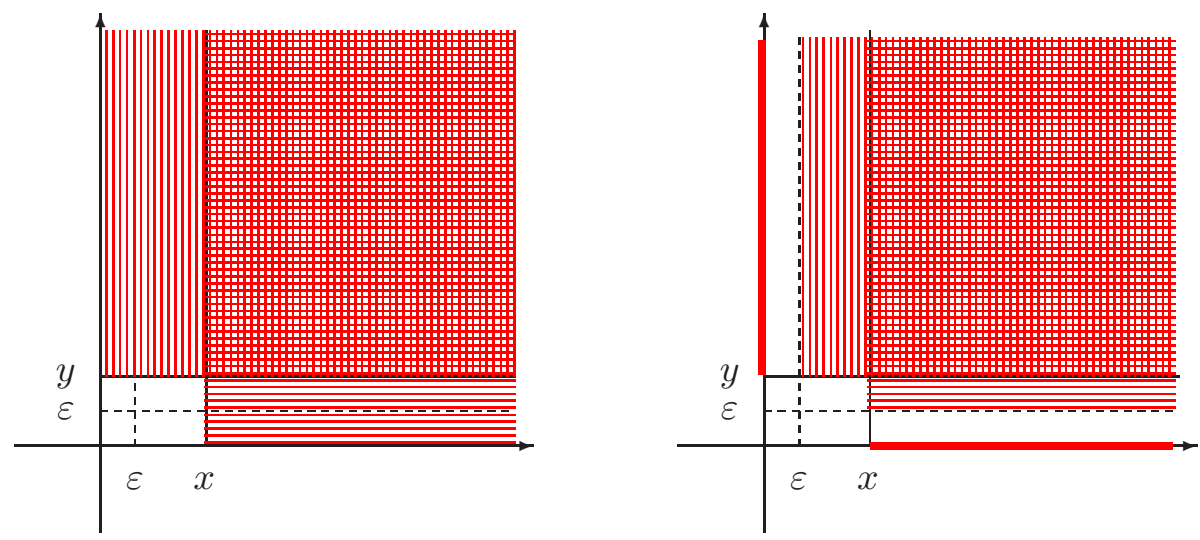

Figure 1: Illustration of the tail integral $\bar{\Pi}$ of a truncated bivariate Lévy process at $(x, y)$ for a process with jump sizes of $\max \{x, y\} \geq \varepsilon$ (left) and a process with jump sizes of $x \geq \varepsilon$ and $y \geq \varepsilon$ (right). Note that in the right plot the mass, where only one component is larger than $\varepsilon>0$ and the other smaller, is projected to the axes.

\section{The observation scheme}

It is based on all jumps of the process larger than some $\varepsilon$ componentwise within the observation interval $[0, t]$. That is, we may observe a single jump $x$ or $y$ either in the first or in the second component. The other observed jumps are $(x, y)$, where $x \geq \varepsilon$ and $y \geq \varepsilon$ at the same time. Let $n=n_{1}+n_{2}$ denote the total number of jumps occurring in $[0, t]$ in either component, where we denote by $n_{1}$ and $n_{2}$ the number of jumps in each marginal process, respectively. This means that we count every joint jump in both components as two jumps. Then $n$ decomposes in the number $n_{1}^{\perp}$ of jumps occurring only in the first component, the number $n_{2}^{\perp}$ of jumps occurring only in the second component, and the number $2 n^{\|}$of jumps occurring in both components.

We denote by $\left(x_{1}, \ldots, x_{n_{1}}, y_{1}, \ldots, y_{n_{2}}\right)$ the observed jumps. By the independence property of the jumps of a Lévy process the order does not matter as long as concurrent jumps remain in the same coordinate. Consequently, throughout the paper we place w.l.o.g. all joint jumps at the beginning of the $x$ - and $y$-observations so that $\left(\mathbf{x}^{\|}, \mathbf{y}^{\|}\right)=\left(\left(x_{1}, y_{1}\right), \ldots,\left(x_{n \|}, y_{n} \|\right)\right)$.

The resulting $n^{\|}+n_{1}^{\perp}+n_{2}^{\perp}$ observations can be attributed to a bivariate CPP similar to the model considered in Esmaeili and Klüppelberg [6]. We shall need the marginal truncated Lévy measures $\Pi_{k}^{(\varepsilon)}$ for $k=1,2$. They will be calculated by first determining the Lévy measures of those processes representing joint jumps larger than $\varepsilon$, denoted by $\Pi^{(\varepsilon) \|}$, single jumps larger than $\varepsilon$ in the first or second component, denoted by $\Pi_{1}^{(\varepsilon) \perp}$ and $\Pi_{2}^{(\varepsilon) \perp}$, respectively.

The tail integrals of the observed CPP are given for $x, y>\varepsilon$ by

$$
\begin{aligned}
& \bar{\Pi}^{(\varepsilon) \|}(x, y)=\bar{\Pi}(x, y), \\
& \bar{\Pi}_{1}{ }^{(\varepsilon) \perp}(x)=\bar{\Pi}(x, 0)-\bar{\Pi}(x, \varepsilon), \\
& \bar{\Pi}_{2}^{(\varepsilon) \perp}(y)=\bar{\Pi}(0, y)-\bar{\Pi}(\varepsilon, y) \text {. }
\end{aligned}
$$


The jump intensities of these CPPs are

$$
\begin{aligned}
\lambda^{(\varepsilon) \|} & =\bar{\Pi}(\varepsilon, \varepsilon), \\
\lambda_{1}{ }^{(\varepsilon) \perp} & =\bar{\Pi}(\varepsilon, 0)-\bar{\Pi}(\varepsilon, \varepsilon), \\
\lambda_{2}{ }^{(\varepsilon) \perp} & =\bar{\Pi}(0, \varepsilon)-\bar{\Pi}(\varepsilon, \varepsilon) .
\end{aligned}
$$

The corresponding jump size distributions are given by the Lévy measures divided by the intensities, respectively. The marginal tail integrals of the truncated processes are now calculated as

$$
\begin{aligned}
& \bar{\Pi}_{1}^{(\varepsilon)}(x)=\bar{\Pi}^{(\varepsilon) \|}(x, \varepsilon)+\bar{\Pi}_{1}^{(\varepsilon) \perp}(x)=\bar{\Pi}(x, 0), \quad x \geq \varepsilon \\
& \bar{\Pi}_{2}^{(\varepsilon)}(y)=\bar{\Pi}^{(\varepsilon) \|}(\varepsilon, y)+\bar{\Pi}_{2}^{(\varepsilon) \perp}(y)=\bar{\Pi}(0, y), \quad y \geq \varepsilon,
\end{aligned}
$$

which implies intensities $\lambda_{k}^{(\varepsilon)}=\lambda^{(\varepsilon) \|}+\lambda_{k}^{(\varepsilon) \perp}=\bar{\Pi}_{k}(\varepsilon)$.

Lemma 4.1 in Esmaeili and Klüppelberg [7] explains the consequence of the small jumps truncation to the Lévy copula. We shall need the notion of a generalized inverse function: for $g: \mathbb{R} \rightarrow \mathbb{R}$ increasing define the generalized inverse of $g$ as $g^{\leftarrow}(x)=\inf \{u \in \mathbb{R}: g(u) \geq x\}$. The definition extends naturally to other supports. For more details and properties of the generalized inverse we refer to Resnick [12, Section 0.2.

From Lemma 4.1 in Esmaeili and Klüppelberg [7] the Lévy copula of the CPP is given by

$$
\mathfrak{C}^{(\varepsilon)}(u, v)=\mathfrak{C}\left(\mathfrak{C}_{1}^{\leftarrow}\left(u, \lambda_{2}^{(\varepsilon)}\right), \mathfrak{C}_{2}^{\leftarrow}\left(\lambda_{1}^{(\varepsilon)}, v\right)\right), \quad 0<u, v<\lambda^{(\varepsilon) \|}
$$

where for $k=1,2$ the symbol $\mathfrak{C}_{k}^{\leftarrow}$ denotes the generalized inverse of $\mathfrak{C}$ with respect to the $k$-th argument.

The following will be our prominent example.

Example 2.2. [Bivariate $\alpha$-stable Clayton subordinator]

Let $c_{1}, c_{2}>0$ and $0<\alpha_{1}, \alpha_{2}<1$. Assume that $\bar{\Pi}_{1}(x)=c_{1} x^{-\alpha_{1}}$ for $x>0$ and $\bar{\Pi}_{2}(y)=c_{2} y^{-\alpha_{2}}$ for $y>0$ and that dependence is modeled by a Clayton Lévy copula, which is given by

$$
\mathfrak{C}(u, v)=\left(u^{-\delta}+v^{-\delta}\right)^{-1 / \delta}, \quad u, v>0
$$

with dependence parameter $\delta>0$.

By (2.3) the tail integrals of the observed CPP are given by

$$
\begin{aligned}
\bar{\Pi}^{(\varepsilon)}(x, y) & =\left(\left(c_{1} x^{-\alpha_{1}}\right)^{-\delta}+\left(c_{2} y^{-\alpha_{2}}\right)^{-\delta}\right)^{-\frac{1}{\delta}}, \quad x, y \geq \varepsilon, \\
\bar{\Pi}_{1}^{(\varepsilon)^{\perp}}(x) & =c_{1} x^{-\alpha_{1}}\left[1-\left(1+\left(\frac{c_{2} \varepsilon^{-\alpha_{2}}}{c_{1} x^{-\alpha_{1}}}\right)^{-\delta}\right)^{-1 / \delta}\right], \quad x \geq \varepsilon, \\
\bar{\Pi}_{2}^{(\varepsilon)^{\perp}}(y) & =c_{2} y^{-\alpha_{2}}\left[1-\left(1+\left(\frac{c_{1} \varepsilon^{-\alpha_{1}}}{c_{2} y^{-\alpha_{2}}}\right)^{-\delta}\right)^{-1 / \delta}\right], \quad y \geq \varepsilon .
\end{aligned}
$$


From (2.4) we calculate the jump intensities

$$
\begin{aligned}
\lambda^{(\varepsilon) \|} & =\left(\left(c_{1} \varepsilon^{-\alpha_{1}}\right)^{-\delta}+\left(c_{2} \varepsilon^{-\alpha_{2}}\right)^{-\delta}\right)^{-\frac{1}{\delta}}, \\
\lambda_{1}^{(\varepsilon)^{\perp}} & =c_{1} \varepsilon^{-\alpha_{1}}\left[1-\left(1+\left(\frac{c_{2} \varepsilon^{-\alpha_{2}}}{c_{1} \varepsilon^{-\alpha_{1}}}\right)^{-\delta}\right)^{-1 / \delta}\right], \\
\lambda_{2}^{(\varepsilon)^{\perp}} & =c_{2} \varepsilon^{-\alpha_{2}}\left[1-\left(1+\left(\frac{c_{1} \varepsilon^{-\alpha_{1}}}{c_{2} \varepsilon^{-\alpha_{2}}}\right)^{-\delta}\right)^{-1 / \delta}\right] .
\end{aligned}
$$

The marginal tail integrals and intensities of the truncated process are now calculated for $k=1,2$ as

$$
\bar{\Pi}_{k}^{(\varepsilon)}(x)=c_{k} x^{-\alpha_{k}}, \quad x \geq \varepsilon, \quad \text { and } \quad \lambda_{k}^{(\varepsilon)}=c_{k} \varepsilon^{-\alpha_{k}} .
$$

This implies for the marginal jump size distributions

$$
\begin{aligned}
P(X>x) & =\bar{\Pi}_{1}^{(\varepsilon)}(x) / \lambda_{1}^{(\varepsilon)}=\varepsilon^{\alpha_{1}} x^{-\alpha_{1}}, \quad x \geq \varepsilon, \\
P(Y>y) & =\bar{\Pi}_{2}^{(\varepsilon)}(y) / \lambda_{2}^{(\varepsilon)}=\varepsilon^{\alpha_{2}} y^{-\alpha_{2}}, \quad y \geq \varepsilon .
\end{aligned}
$$

By (2.5) the Lévy copula of the observed CPP is for $0<u, v<\lambda^{(\varepsilon) \|}$ given by

$$
\begin{aligned}
\mathfrak{C}^{(\varepsilon)}(u, v) & =\mathfrak{C}\left(\left(u^{-\delta}-\left(\lambda_{2}^{(\varepsilon)}\right)^{-\delta}\right)^{-1 / \delta},\left(v^{-\delta}-\left(\lambda_{1}^{(\varepsilon)}\right)^{-\delta}\right)^{-1 / \delta}\right) \\
& =\left(u^{-\delta}+v^{-\delta}-\left(c_{1}^{-\delta} \varepsilon^{\alpha_{1} \delta}+c_{2}^{-\delta} \varepsilon^{\alpha_{2} \delta}\right)\right)^{-1 / \delta} .
\end{aligned}
$$

\section{Two-step parameter estimation of a Lévy process}

The idea of a two-step procedure for subordinators is similar to the IFM method for multivariate distributions. The term IFM is the acronym for "inference functions for margins" and has been applied in various areas of multivariate statistics; cf. Godambe [8] and Joe [10], Ch. 10. Obviously, the maximization of a likelihood with many parameters can be numerically sophisticated and computationally time-consuming; in a two-step method the parameters of the marginal components are estimated first and the Lévy copula parameters in a second step, thus reducing the dimensionality of the problem. For multivariate distribution functions, the algorithm is explained, for instance, in Joe [10], Ch. 10.

For a multivariate Lévy process in $\mathbb{R}^{d}$ for arbitrary dimension $d \in \mathbb{N}$, the two-step algorithm can be formalized as follows.

Step 1 : We do not distinguish between single and common jumps, but make use of all data available; i.e., we take all observations $x_{i k}>\varepsilon$ for $i=1, \ldots, n_{k}$ and all $k=1, \ldots, d$. We denote by $\gamma=\left(\theta_{1}, \ldots, \theta_{d}\right)$ the vector of all marginal parameters (the $\theta_{i}$ are usually vectors) and let

$l_{1}^{(\varepsilon)}, \ldots, l_{d}^{(\varepsilon)}$ be the marginal log-likelihood functions with respect to the parameters. Determine

$$
\widetilde{\gamma}:=\operatorname{argmax}_{\gamma} \sum_{k=1}^{d} l_{k}^{(\varepsilon)}\left(\theta_{k} \mid \mathbf{x}_{k}\right),
$$


where $\mathbf{x}_{k}=\left(x_{1 k}, x_{2 k}, \ldots, x_{n_{k} k}\right)$ are all observations in component $k$ larger than $\varepsilon$.

Step 2 : Write the log-likelihood $l^{(\varepsilon)}$ of a CPP, whose jumps are only the common jumps of $x_{i k}>\varepsilon$ for $i=1, \ldots, n^{\|}$and $k=1, \ldots, d$, plug in the marginal parameter estimates from Step 1, resulting in the log-likelihood of a $\mathrm{CPP}$ with only dependence structure parameter $\delta$. Maximize the $\log$-likelihood function over $\delta$; i.e., estimate the Lévy copula parameter vector $\delta \in \mathbb{R}^{m}$ for some $m \in \mathbb{N}$, based on the common jumps:

$$
\widetilde{\delta}:=\operatorname{argmax}_{\delta} l^{(\varepsilon)}\left(\delta \mid \widetilde{\gamma}, \mathbf{x}_{1}^{\|}, \ldots, \mathbf{x}_{d}^{\|}\right)
$$

where $\widetilde{\gamma}=\left(\widetilde{\theta}_{1}, \ldots, \widetilde{\theta}_{d}\right)$ and $\mathbf{x}_{k}^{\|}=\left(x_{1 k}, \ldots, x_{n \|_{k}}\right)$ for $k=1, \ldots, d$.

Remark 3.1. The MLE $\hat{\eta}$ of the parameter vector $\eta=\left(\theta_{1}, \ldots, \theta_{d}, \delta\right)$ is derived by maximization of the log-likelihood of the multivariate $\operatorname{CPP} l^{(\varepsilon)}$ over the parameter vector $\eta$ (as done in [7]). The estimate $\hat{\eta}$ is the solution of

$$
\left(\frac{\partial l^{(\varepsilon)}}{\partial \theta_{1}}, \ldots, \frac{\partial l^{(\varepsilon)}}{\partial \theta_{d}}, \frac{\partial l^{(\varepsilon)}}{\partial \delta}\right)=0 .
$$

This is in contrast with the two-step method, where the estimate $\widetilde{\eta}$ is the solution of

$$
\left(\frac{\partial l_{1}^{(\varepsilon)}}{\partial \theta_{1}}, \ldots, \frac{\partial l_{k}^{(\varepsilon)}}{\partial \theta_{k}}, \frac{\partial l^{(\varepsilon)}}{\partial \delta}\right)=0 .
$$

Remark 3.2. The aim of the two-step method is in fact to reduce the dimension of the parameter vector to have a simpler structure for the optimization of the likelihood function. Note that the observation scheme in [7], which takes only the $n^{\|}$observations of the joint jumps in both steps into account, fails this goal, since the observation scheme used there introduces the dependence parameter into the marginal likelihoods.

\subsection{Two-step estimation method of an $\alpha$-stable Clayton subordinator with different marginal parameters}

The following algorithm works in principle in every dimension. For notational simplicity we formulate it only for dimension $d=2$. Let $\mathbf{S}=\left(S_{1}, S_{2}\right)$ be a bivariate $\alpha$-stable Clayton subordinator as introduced in Example 2.2 with different marginal parameters $\theta_{1}=\left(\alpha_{1}, c_{1}\right)$ and $\theta_{2}=\left(\alpha_{2}, c_{2}\right)$ with $\alpha_{k} \in(0,1)$ and $c_{k} \in(0, \infty)$ for $k=1,2$ and a Lévy copula parameter $\delta \in(0, \infty)$. We assume the observation scheme as described in Section 2 . We denote by $\left(X_{1}, \ldots, X_{n \|}, \ldots, X_{n_{1}}, Y_{1}, \ldots, Y_{n \|}, \ldots, Y_{n_{2}}\right)$ the vector of jumps larger than $\varepsilon$ for the component processes $S_{1}^{(\varepsilon)}$ and $S_{2}^{(\varepsilon)}$, respectively. As before, all double jumps are numbered as $\left(X_{i}, Y_{i}\right)$ for $i=1, \ldots, n^{\|}$.

Step 1 : Since the marginal log-likelihoods have the same structure with no common parameters, 3.1) decomposes in its components for $S_{1}$ and $S_{2}$, and maximization is done separately. We proceed as in Basawa and Brockwell [1, 2]; cf. Esmaeili and Klüppelberg [7], Example 3.1, 
and exemplify it for the first component:

$$
l_{1}^{(\varepsilon)}\left(\log c_{1}, \alpha_{1} ; \mathbf{x}\right)=-c_{1} t \varepsilon^{-\alpha_{1}}+n_{1}\left(\log c_{1}+\log \alpha_{1}\right)-\left(\alpha_{1}+1\right) \sum_{i=1}^{n_{1}} \log x_{i} .
$$

From Basawa and Brockwell [1, 2] we know that the marginal MLEs of $c_{1}$ and $\alpha_{1}$ and the intensity parameter $\lambda_{1}^{(\varepsilon)}$ are given by

$$
\begin{aligned}
\widetilde{\lambda}_{1}^{(\varepsilon)} & =\frac{n_{1}}{t} \\
\widetilde{\alpha}_{1} & =\left(\frac{1}{n_{1}} \sum_{i=1}^{n_{1}}\left(\log X_{i}-\log \varepsilon\right)+\log \varepsilon\left(1-\frac{\lambda_{1}^{(\varepsilon)}}{\widetilde{\lambda}_{1}^{(\varepsilon)}}\right)\right)^{-1}, \\
\log \widetilde{c}_{1} & =\log \widetilde{\lambda}_{1}^{(\varepsilon)}+\widetilde{\alpha}_{1} \log \varepsilon .
\end{aligned}
$$

Furthermore, asymptotic normality holds with degenerate limit for $\left(\widetilde{c}_{1}, \widetilde{\alpha}_{1}\right)$ and with asymptotic independence for $\left(\widetilde{\lambda}_{1}, \widetilde{\alpha}_{1}\right)$ as $n_{1} \rightarrow \infty$. Limit laws hold for both situations, $t \rightarrow \infty$ or $\varepsilon \rightarrow 0$. The first limit was derived in Basawa and Brockwell [2]. Asymptotic independence for the second vector was shown in Höpfner and Jacod [9]. Both results are reported with precise rates and the asymptotic covariance matrix in Esmaeili and Klüppelberg [7, Example 3.1.

Step 2 : We first determine the log-likelihood function in (3.2) for the bivariate CPP of common jumps larger than $\varepsilon$. By (2.7) the intensity is $\lambda^{(\varepsilon) \|}=\left(c_{1}^{-\delta} \varepsilon^{\alpha_{1} \delta}+c_{2}^{-\delta} \varepsilon^{\alpha_{2} \delta}\right)^{-\frac{1}{\delta}}$. Together with (2.6) this yields the survival function of bivariate joint jumps

$$
\bar{F}^{(\varepsilon)}(x, y)=\left(\frac{c_{1}^{-\delta} x^{\alpha_{1} \delta}+c_{2}^{-\delta} y^{\alpha_{2} \delta}}{c_{1}^{-\delta} \varepsilon^{\alpha_{1} \delta}+c_{2}^{-\delta} \varepsilon^{\alpha_{2} \delta}}\right)^{-\frac{1}{\delta}}, \quad x, y \geq \varepsilon,
$$

with density given by

$$
f^{(\varepsilon)}(x, y)=\frac{\alpha_{1} \alpha_{2}(1+\delta)\left(c_{1}^{-\delta} \varepsilon^{\alpha_{1} \delta}+c_{2}^{-\delta} \varepsilon^{\alpha_{2} \delta}\right)^{\frac{1}{\delta}}}{\left(c_{1} c_{2}\right)^{\delta}} \frac{x^{\alpha_{1} \delta-1} y^{\alpha_{2} \delta-1}}{\left(c_{1}^{-\delta} x^{\alpha_{1} \delta}+c_{2}^{-\delta} y^{\alpha_{2} \delta}\right)^{\frac{1}{\delta}+2}} .
$$

This results in the log-likelihood function

$$
\begin{aligned}
& l^{(\varepsilon)}\left(c_{1}, c_{2}, \alpha_{1}, \alpha_{2}, \delta ; \mathbf{x}^{\|}, \mathbf{y}^{\|}\right)=-\lambda^{(\varepsilon)} \|_{t}+n^{\|} \log (1+\delta)-n^{\|} \delta\left(\log c_{1}+\log c_{2}\right) \\
& +n^{\|}\left(\log \alpha_{1}+\log \alpha_{2}\right)+\left(\alpha_{1} \delta-1\right) \sum_{i=1}^{n^{\|}} \log x_{i}+\left(\alpha_{2} \delta-1\right) \sum_{i=1}^{n^{\|}} \log y_{i} \\
& -\left(\frac{1}{\delta}+2\right) \sum_{i=1}^{n^{\|}} \log \left(c_{1}^{-\delta} x_{i}^{\alpha_{1} \delta}+c_{2}^{-\delta} y_{i}^{\alpha_{2} \delta}\right),
\end{aligned}
$$

where $\left(\mathbf{x}^{\|}, \mathbf{y}^{\|}\right)=\left(\left(x_{1}, y_{1}\right), \ldots,\left(x_{n}\left\|, y_{n}\right\|\right)\right)$.

Given the marginal parameter estimates from the first step, the score function with respect 
to the dependence parameter $\delta$ is given by

$$
\begin{aligned}
& \frac{\partial l^{(\varepsilon)}\left(\delta \mid \widetilde{\gamma}, \mathbf{x}^{\|}, \mathbf{y}^{\|}\right)}{\partial \delta}=-\frac{\partial \lambda^{(\varepsilon) \|}}{\partial \delta} t+\frac{n^{\|}}{1+\delta}-n^{\|}\left(\log \widetilde{c}_{1}+\log \widetilde{c}_{2}\right) \\
& +\widetilde{\alpha}_{1} \sum_{i=1}^{n^{\|}} \log x_{i}+\widetilde{\alpha}_{2} \sum_{i=1}^{n^{\|}} \log y_{i}+\frac{1}{\delta^{2}} \sum_{i=1}^{n^{\|}} \log \left(\widetilde{c}_{1}^{-\delta} x_{i}^{\widetilde{\alpha}_{1} \delta}+\widetilde{c}_{2}^{-\delta} y_{i}^{\widetilde{\alpha}_{2} \delta}\right) \\
& -\left(\frac{1}{\delta}+2\right) \sum_{i=1}^{n^{\|}} \frac{\partial}{\partial \delta} \log \left(\widetilde{c}_{1}^{-\delta} x_{i}^{\widetilde{\alpha}_{1} \delta}+\widetilde{c}_{2}^{-\delta} y_{i}^{\widetilde{\alpha}_{2} \delta}\right) .
\end{aligned}
$$

The parameter estimate $\widetilde{\delta}$ can be found numerically by solving the following equation for $\delta$ :

$$
\frac{\partial l^{(\varepsilon)}\left(\delta \mid \widetilde{\gamma}, \mathbf{x}^{\|}, \mathbf{y}^{\|}\right)}{\partial \delta}=0
$$

Remark 3.3. The vector of score functions in the two-step method is given by

$$
\begin{gathered}
\mathbf{J}^{(\varepsilon)}(\mathbf{X}, \mathbf{Y} ; \eta)= \\
\left(\frac{\partial l_{1}^{(\varepsilon)}\left(\log c_{1}, \alpha_{1} ; \mathbf{X}\right)}{\partial \log c_{1}}, \frac{\partial l_{1}^{(\varepsilon)}\left(\log c_{1}, \alpha_{1} ; \mathbf{X}\right)}{\partial \alpha_{1}}, \frac{\partial l_{2}^{(\varepsilon)}\left(\log c_{2}, \alpha_{2} ; \mathbf{Y}\right)}{\partial \log c_{2}}, \frac{\partial l_{2}^{(\varepsilon)}\left(\log c_{2}, \alpha_{2} ; \mathbf{Y}\right)}{\partial \alpha_{2}}, \frac{\partial l^{(\varepsilon)}\left(\delta ; \mathbf{X}^{\|}, \mathbf{Y}^{\|}\right)}{\partial \delta}\right)^{T}
\end{gathered}
$$

where $\eta=\left(\log c_{1}, \log c_{2}, \alpha_{1}, \alpha_{2}, \delta\right)^{T}$ is the parameter vector, $\mathbf{X}=\left(X_{1}, \ldots, X_{n_{1}}\right), \mathbf{Y}=\left(Y_{1}, \ldots, Y_{n_{2}}\right)$ and $\left(\mathbf{X}^{\|}, \mathbf{Y}^{\|}\right)=\left(\left(X_{1}, Y_{1}\right), \ldots,\left(X_{n \|}, Y_{n \|}\right)\right)$.

\subsection{Two-step method for a bivariate $\alpha$-stable Clayton subordinator with com- mon marginal parameters}

For an analysis of the two-step estimation procedure we simplify the model as follows. Let $\mathbf{S}=\left(S_{1}, S_{2}\right)$ be a bivariate $\alpha$-stable subordinator as in Example 2.2 with common marginal parameters $\theta_{1}=\theta_{2}=(\alpha, c)$ and a Clayton Lévy copula parameter $\delta$. Assume an observation scheme as explained in Section 2, Maximum likelihood estimation for the parameters of this model was discussed in Esmaeili and Klüppelberg [7] in detail. In this section we estimate the parameters with the two-step method.

Step 1 : The log-likelihood function (3.1), which ignores the dependence structure, is given by

$$
\begin{aligned}
l_{12}^{(\varepsilon)}(\log c, \alpha) & =l_{1}^{(\varepsilon)}(\log c, \alpha)+l_{2}^{(\varepsilon)}(\log c, \alpha) \\
& =-2 c t \varepsilon^{-\alpha}+n(\log c+\log \alpha)-(\alpha+1) \sum_{i=1}^{n} \log z_{i}
\end{aligned}
$$

where $n:=n_{1}+n_{2}$ is Poisson distributed. Since $n_{1}$ and $n_{2}$ have both intensity $\lambda^{(\varepsilon)}:=\lambda_{1}^{(\varepsilon)}=$ $\lambda_{2}^{(\varepsilon)}, n$ has intensity $2 \lambda^{(\varepsilon)}=2 c \varepsilon^{-\alpha}$ and $\left(z_{1}, \ldots, z_{n}\right)=\left(x_{1}, \ldots, x_{n_{1}}, y_{1}, \ldots, y_{n_{2}}\right)$. Note that the corresponding random variables $\log \left(\frac{Z_{i}}{\varepsilon}\right)$, for $i=1, \ldots, n$ are exponentially distributed with density $f(u)=\alpha e^{-\alpha u}$ for $u>0$. The log-likelihood has score functions with respect to the 
marginal parameters $\log c$ and $\alpha$ as follows:

$$
\begin{aligned}
& \frac{\partial l_{12}^{(\varepsilon)}(\log c, \alpha)}{\partial \log c}=n-2 c t \varepsilon^{-\alpha}=n-2 \lambda^{(\varepsilon)} t \\
& \frac{\partial l_{12}^{(\varepsilon)}(\log c, \alpha)}{\partial \alpha}=\frac{n}{\alpha}+2 c t \varepsilon^{-\alpha} \log \varepsilon-\sum_{i=1}^{n} \log z_{i}=-\sum_{i=1}^{n}\left(\log \frac{z_{i}}{\varepsilon}-\frac{1}{\alpha}\right)-\left(n-2 \lambda^{(\varepsilon)} t\right) \log \varepsilon .
\end{aligned}
$$

The common intensity parameter $\lambda^{(\varepsilon)}=c \varepsilon^{-\alpha}$ and the marginal parameters $\log c$ and $\alpha$ can be estimated by (3.3) as

$$
\begin{aligned}
\widetilde{\lambda}^{(\varepsilon)} & =\frac{n}{2 t} \\
\widetilde{\alpha} & =\left(\frac{1}{n} \sum_{i=1}^{n}\left(\log Z_{i}-\log \varepsilon\right)+\left(1-\frac{\lambda^{(\varepsilon)}}{\widetilde{\lambda}^{(\varepsilon)}}\right) \log \varepsilon\right)^{-1} \\
\log \widetilde{c} & =\log \widetilde{\lambda}^{(\varepsilon)}+\widetilde{\alpha} \log \varepsilon .
\end{aligned}
$$

Step 2 : As explained in Esmaeili and Klüppelberg [7, for simplifying the calculations of the second derivatives later we reparameterize the dependence to $\theta=\alpha \delta$. The joint density of bivariate jumps is a special case of (3.4) and has been calculated in (4.10) in Esmaeili and Klüppelberg [7]. From (2.7) we know that $\lambda^{(\varepsilon) \|}=c \varepsilon^{-\alpha} 2^{-\frac{\alpha}{\theta}}$, which we use for abbreviation. Then the log-likelihood in (3.2) is

$$
\begin{aligned}
& l^{(\varepsilon)}(\log c, \alpha, \theta)=-\lambda^{(\varepsilon)} \|_{t+n^{\|}} \log \alpha+n^{\|} \log (\alpha+\theta)+n^{\|} \log c \\
& +(\theta-1) \sum_{i=1}^{n \|}\left(\log x_{i}+\log y_{i}\right)-\left(2+\frac{\alpha}{\theta}\right) \sum_{i=1}^{n^{\|}} \log \left(x_{i}^{\theta}+y_{i}^{\theta}\right) \text {. }
\end{aligned}
$$

The score function with respect to the parameter $\theta$ is then given by (the derivatives of $\lambda^{(\varepsilon) \|}$ are calculated in Lemma 4.1 below)

$$
\begin{aligned}
\frac{\partial l^{(\varepsilon)}}{\partial \theta}= & -\frac{\partial \lambda^{(\varepsilon) \|}}{\partial \theta} t+\frac{n^{\|}}{\alpha+\theta}+\sum_{i=1}^{n^{\|}}\left(\log x_{i}+\log y_{i}\right) \\
& +\frac{\alpha}{\theta^{2}} \sum_{i=1}^{n^{\|}} \log \left(x_{i}^{\theta}+y_{i}^{\theta}\right)-\left(2+\frac{\alpha}{\theta}\right) \sum_{i=1}^{n^{\|}} \frac{\partial}{\partial \theta} \log \left(x_{i}^{\theta}+y_{i}^{\theta}\right) .
\end{aligned}
$$

Given the estimates of the marginal parameters $\widetilde{c}$ and $\widetilde{\alpha}$ from the first step, the estimate of $\theta$ can be computed numerically as the argmax of the right hand side of (3.8).

Remark 3.4. The vector of score functions from Remark 3.3 reduces to

$$
\mathbf{J}^{(\varepsilon)}(\mathbf{X}, \mathbf{Y} ; \eta)=\left(\frac{\partial l_{12}^{(\varepsilon)}(\log c, \alpha ; \mathbf{Z})}{\partial \log c}, \frac{\partial l_{12}^{(\varepsilon)}(\log c, \alpha ; \mathbf{Z})}{\partial \alpha}, \frac{\partial l^{(\varepsilon)}\left(\log c, \alpha, \theta ; \mathbf{X}^{\|}, \mathbf{Y}^{\|}\right)}{\partial \theta}\right)^{T}
$$

where $\eta=(\log c, \alpha, \theta)^{T}$ is the parameter vector, $\mathbf{Z}=\left(X_{1}, \ldots, X_{n_{1}}, Y_{1}, \ldots, Y_{n_{2}}\right)$ and $\left(\mathbf{X}^{\|}, \mathbf{Y}^{\|}\right)=$ $\left(X_{1}, Y_{1}\right), \ldots,\left(X_{n \|}, Y_{n \|}\right)$. 


\section{Asymptotic properties of the two-step estimates}

The two-step estimation procedure is a special case of the estimating functions approach, which goes back to Godambe [8] (see also the Z estimates in van der Vaart [14]). In this framework, the Godambe information matrix plays the role of the Fisher information matrix in classical MLE.

We explain this for Lévy copulas. Let $\mathbf{S}=\left(S_{1}, S_{2}\right)$ be a bivariate $\alpha$-stable Clayton subordinator with parameter vector $\eta \in \mathbb{R}^{k}$ including marginal and dependence parameters. Assume further an observation scheme as explained in Section 2, In principle the two-step estimation procedure can be applied to both situations of Section 3.1 with $\eta \in \mathbb{R}^{5}$ or of Section 3.2 with $\eta \in \mathbb{R}^{3}$.

For the vector of score functions, denoted by $\mathbf{J}^{(\varepsilon)}(\mathbf{X}, \mathbf{Y} ; \eta)$ as in Remarks 3.3 and 3.4 , the so-called Godambe information matrix is calculated for fixed $\varepsilon>0$ as

$$
G:=D^{\top} M^{-1} D
$$

where

$$
\begin{aligned}
D & :=\frac{1}{2 \lambda^{(\varepsilon)} t} \mathbb{E}\left[-\frac{\partial \mathbf{J}^{(\varepsilon)}(\mathbf{X}, \mathbf{Y} ; \eta)}{\partial \eta}\right], \\
M & :=\frac{1}{2 \lambda^{(\varepsilon)} t} \mathbb{E}\left[\mathbf{J}^{(\varepsilon)}(\mathbf{X}, \mathbf{Y} ; \eta) \mathbf{J}^{(\varepsilon)}(\mathbf{X}, \mathbf{Y} ; \eta)^{T}\right]
\end{aligned}
$$

are $k \times k$-matrices. Under appropriate conditions, which will be shown below, the asymptotic covariance matrix of $n^{-\frac{1}{2}}(\widetilde{\eta}-\eta)$ is equal to the inverse of $G$.

For the remainder of this section we restrict the process $\mathbf{S}$ again to the model in Section 3.2 , a bivariate $\alpha$-stable subordinator with common marginal parameters $\log c$ and $\alpha$, and dependence parameter $\theta$. We denote by $l_{12}^{(\varepsilon)}$ the log-likelihood of the common marginal parameters $\gamma:=$ $(\log c, \alpha)$ as in (3.5), and by $l^{(\varepsilon)}$ the $\log$-likelihood of the bivariate CPP in the second step as in (3.7). Assume further that $\eta_{0}=\left(\log c_{0}, \alpha_{0}, \theta_{0}\right)$ is the true parameter vector. We prove consistency of the two-step estimators, and their joint asymptotic normality. We calculate the Godambe information matrix $G$ as well as the asymptotic covariance matrix of the estimators.

There is a fundamental difference between our approach and the classical used for distributional copulas in Joe [10, Section 10.1.1. Whereas he can work with a fixed number of multivariate data, our process structure with observations on an interval $[0, t]$ implies a random number of data points. Moreover, we have to deal with the problem of single and common jumps. Furthermore, [10] assumes regularity conditions like interchangeability of derivatives and integrals, which are not necessarily guaranteed in our context (cf. Esmaeili and Klüppelberg [7], Section 4.2). As a consequence, we will provide a full proof of the asymptotic normality of the IFM estimators in Theorem 4.8 below.

\subsection{Auxiliary results}

We shall need the following derivatives of $\lambda^{(\varepsilon) \|}$. 
Lemma 4.1. For $\lambda^{(\varepsilon) \|}=c \varepsilon^{-\alpha} 2^{-\frac{\alpha}{\theta}}$ the partial derivatives are given by

$$
\frac{\partial \lambda^{(\varepsilon) \|}}{\partial \log c}=\lambda^{(\varepsilon) \|}, \quad \frac{\partial \lambda^{(\varepsilon) \|}}{\partial \alpha}=-\lambda^{(\varepsilon) \|}\left(\log \varepsilon+\frac{1}{\theta} \log 2\right), \quad \frac{\partial \lambda^{(\varepsilon) \|}}{\partial \theta}=\lambda^{(\varepsilon) \|} \frac{\alpha \log 2}{\theta^{2}} .
$$

The second derivatives can be calculated as

$$
\begin{aligned}
\frac{\partial^{2} \lambda^{(\varepsilon) \|}}{\partial \theta \partial \log c} & =\lambda^{(\varepsilon) \|} \frac{\alpha \log 2}{\theta^{2}}, \\
\frac{\partial^{2} \lambda^{(\varepsilon) \|}}{\partial \theta \partial \alpha} & =-\lambda^{(\varepsilon) \|} \frac{\log 2}{\theta^{2}}\left(\alpha \log \varepsilon+\frac{\alpha}{\theta} \log 2-1\right), \\
\frac{\partial^{2} \lambda^{(\varepsilon) \|}}{\partial \theta^{2}} & =\lambda^{(\varepsilon) \|} \frac{\alpha \log 2}{\theta^{2}}\left(\frac{\alpha \log 2}{\theta^{2}}-\frac{2}{\theta}\right) .
\end{aligned}
$$

We calculate several matrices for later use, where the details are given in the Appendix. Throughout we abbreviate $d=\frac{\lambda^{(\varepsilon) \|}}{2 \lambda^{(\varepsilon)}}=2^{-\frac{\alpha}{\theta}-1}$.

Lemma 4.2. We denote by $H^{(\varepsilon)}=\frac{\partial \mathbf{J}^{(\varepsilon)}(\mathbf{X}, \mathbf{Y} ; \eta)}{\partial \eta}$ the matrix of the second-order derivatives of the two-step log-likelihood functions (3.5) and (3.7), respectively. Then

$$
H^{(\varepsilon)}=2 \lambda^{(\varepsilon)} t\left(\begin{array}{ccc}
-1 & \log \varepsilon & 0 \\
\log \varepsilon & -\frac{n}{\alpha^{2} 2 \lambda^{(\varepsilon)} t}-(\log \varepsilon)^{2} & 0 \\
-d \frac{\alpha \log 2}{\theta^{2}} & d\left(\frac{\alpha \log 2}{\theta^{2}} \log \varepsilon-A\right) & -d B
\end{array}\right)
$$

where

$$
\begin{aligned}
A(\eta):= & -\frac{\alpha(\log 2)^{2}}{\theta^{3}}+\frac{\log 2}{\theta^{2}}+\frac{n^{\|}}{\lambda^{(\varepsilon)} t_{(\alpha+\theta)^{2}}}-\frac{1}{\lambda^{(\varepsilon)} \| t \theta^{2}} \sum_{i=1}^{n^{\|}} \log \left(X_{i}^{\theta}+Y_{i}^{\theta}\right) \\
& +\frac{1}{\lambda^{(\varepsilon)} \|_{t}} \sum_{i=1}^{n^{\|}} \frac{\partial}{\partial \theta} \log \left(X_{i}^{\theta}+Y_{i}^{\theta}\right), \\
B(\eta):= & \left(\frac{\alpha \log 2}{\theta^{2}}\right)^{2}-\frac{2 \alpha \log 2}{\theta^{3}}+\frac{n^{\|}}{\lambda^{(\varepsilon)} t_{t}(\alpha+\theta)^{2}}+\frac{2 \alpha}{\lambda^{(\varepsilon)} \|_{t \theta^{3}}} \sum_{i=1}^{n^{\|}} \log \left(X_{i}^{\theta}+Y_{i}^{\theta}\right) \\
& -\frac{2 \alpha}{\lambda^{(\varepsilon) \|} t \theta^{2}} \sum_{i=1}^{n^{\|}} \frac{\partial}{\partial \theta} \log \left(X_{i}^{\theta}+Y_{i}^{\theta}\right)+\frac{2 \theta+\alpha}{\lambda^{(\varepsilon)} \|_{t \theta}} \sum_{i=1}^{n^{\|}} \frac{\partial^{2}}{\partial \theta^{2}} \log \left(X_{i}^{\theta}+Y_{i}^{\theta}\right) .
\end{aligned}
$$

We present the two matrices $D$ and $M$ from (4.2) and (4.3), respectively.

Lemma 4.3. Assume a bivariate $\alpha$-stable Clayton subordinator with common marginal parameters $(\log c, \alpha)$ and dependence parameter $\theta=\alpha \delta$. Assume also the observation scheme

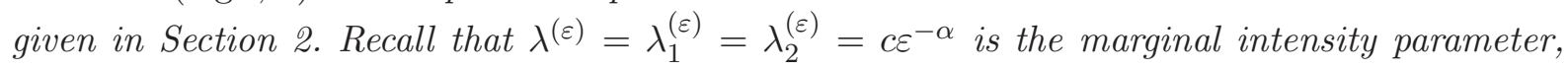


$\lambda^{(\varepsilon) \|}=c \varepsilon^{-\alpha} 2^{-\frac{\alpha}{\theta}}$ is the joint jumps intensity parameter. Then the matrix $D=-\frac{1}{2^{(\varepsilon)} t} \mathbb{E}\left[H^{(\varepsilon)}\right]$ of (4.2) is given by

$$
D=\left(\begin{array}{ccc}
1 & -\log \varepsilon & 0 \\
-\log \varepsilon & \frac{1}{\alpha^{2}}+(\log \varepsilon)^{2} & 0 \\
d \frac{\alpha \log 2}{\theta^{2}} & d\left(-\frac{\alpha \log 2}{\theta^{2}} \log \varepsilon+a\right) & d b
\end{array}\right)
$$

where

$$
\begin{aligned}
a(\alpha, \theta)= & -\frac{\alpha(\log 2)^{2}}{\theta^{3}}+\frac{\log 2}{\theta^{2}}+\frac{1}{(\alpha+\theta)^{2}}-\frac{1}{\theta^{2}} \mathbb{E}\left[\log \left(X_{1}^{\theta}+Y_{1}^{\theta}\right)\right]+\frac{1}{\theta} \mathbb{E}\left[\frac{\partial}{\partial \theta} \log \left(X_{1}^{\theta}+Y_{1}^{\theta}\right)\right] \\
b(\alpha, \theta)= & \left(\frac{\alpha \log 2}{\theta^{2}}\right)^{2}-\frac{2 \alpha \log 2}{\theta^{3}}+\frac{1}{(\alpha+\theta)^{2}}+\frac{2 \alpha}{\theta^{3}} \mathbb{E}\left[\log \left(X_{1}^{\theta}+Y_{1}^{\theta}\right)\right] \\
& -\frac{2 \alpha}{\theta^{2}} \mathbb{E}\left[\frac{\partial}{\partial \theta} \log \left(X_{1}^{\theta}+Y_{1}^{\theta}\right)\right]+\frac{2 \theta+\alpha}{\theta} \mathbb{E}\left[\frac{\partial^{2}}{\partial \theta^{2}} \log \left(X_{1}^{\theta}+Y_{1}^{\theta}\right)\right] .
\end{aligned}
$$

Remark 4.4. (i) The functions $a(\alpha, \theta)$ and $b(\alpha, \theta)$ are deterministic functions of the parameters and do not depend on $t$ or $\varepsilon$. Moreover, all expectations in (4.6) and (4.7) are finite, since $\log X_{i}$ and $\log Y_{i}$ are exponentially distributed (cf. Lemma 4.4 of Esmaeili and Klüppelberg [7].)

(ii) We shall need the following inverse, which exists for $b \neq 0$ :

$$
D^{-1}=\left(\begin{array}{ccc}
1+\alpha^{2}(\log \varepsilon)^{2} & \alpha^{2} \log \varepsilon & 0 \\
\alpha^{2} \log \varepsilon & \alpha^{2} & 0 \\
-\frac{1}{b}\left(a \alpha^{2} \log \varepsilon+\frac{\alpha \log 2}{\theta^{2}}\right) & -\frac{a}{b} \alpha^{2} & \frac{1}{d b}
\end{array}\right) .
$$

In order to calculate the matrix $M$ from (4.3) we shall need the following result on the dependence of $n$ and $n^{\|}$.

Lemma 4.5. Recall that $n=n_{1}+n_{2}=2 n^{\|}+n_{1}^{\perp}+n_{2}^{\perp}$. Then

$$
\mathbb{E}\left[n n^{\|}\right]=2 \lambda^{(\varepsilon) \|} t\left(1+\lambda^{(\varepsilon)} t\right) \quad \text { and } \operatorname{Cov}\left(n, n^{\|}\right)=2 \lambda^{(\varepsilon) \|_{t}} .
$$

Proof. We calculate the expectation, the result for the covariance is then obvious. By independence of the Poisson processes of joint and single jumps,

$$
\begin{aligned}
\mathbb{E}\left[n n^{\|}\right] & =\mathbb{E}\left[\left(2 n^{\|}+n_{1}^{\perp}+n_{2}^{\perp}\right) n^{\|}\right] \\
& =2\left(\operatorname{Var}\left(n^{\|}\right)+\left(\mathbb{E}\left[n^{\|}\right]\right)^{2}\right)+\mathbb{E}\left[n_{1}^{\perp}+n_{2}^{\perp}\right] \mathbb{E}\left[n^{\|}\right] \\
& =2\left(\lambda^{(\varepsilon) \|_{t}} t\left(\lambda^{(\varepsilon) \|} t\right)^{2}\right)+\left(\lambda_{1}^{(\varepsilon) \perp}+\lambda_{2}^{(\varepsilon) \perp}\right) \lambda^{(\varepsilon) \|} t^{2} \\
& =2 \lambda^{(\varepsilon) \|} t\left(1+\lambda^{(\varepsilon)} t\right) .
\end{aligned}
$$


Lemma 4.6. Assume a bivariate $\alpha$-stable Clayton subordinator with common marginal parameters $(\log c, \alpha)$ and dependence parameter $\theta=\alpha \delta$. Assume also the observation scheme given in Section 2. Define

$$
T(x, y):=(\log x+\log y)+\frac{\alpha}{\theta^{2}} \log \left(x^{\theta}+y^{\theta}\right)-\left(2+\frac{\alpha}{\theta}\right) \frac{\partial}{\partial \theta} \log \left(x^{\theta}+y^{\theta}\right) .
$$

Then the matrix $M$ introduced in (4.3) is given by

$$
M=\left(\begin{array}{ccc}
1 & -\log \varepsilon & 2 d \frac{\alpha \log 2}{\theta^{2}} \\
-\log \varepsilon & \frac{1}{\alpha^{2}}+(\log \varepsilon)^{2} & -d\left(2 \frac{\alpha \log 2}{\theta^{2}} \log \varepsilon+m\right) \\
2 d \frac{\alpha \log 2}{\theta^{2}} & -d\left(2 \frac{\alpha \log 2}{\theta^{2}} \log \varepsilon+m\right) & d b
\end{array}\right),
$$

where $b$ is given by (4.7) and

$$
m=2 \operatorname{Cov}\left(\log \frac{X_{1}}{\varepsilon}, T\left(\frac{X_{1}}{\varepsilon}, \frac{Y_{1}}{\varepsilon}\right)\right) .
$$

Moreover, this covariance is independent of $\varepsilon$.

\subsection{Consistency and asymptotic normality of the two-step estimators}

Assume that the $\log$-likelihood $l_{12}^{(\varepsilon)}(\log c, \alpha)$ in (3.5) is used for estimating the marginal parameters $\log c$ and $\alpha$ in the first step and the $\log$-likelihood $l^{(\varepsilon)}(\log c, \alpha, \theta)$ in (3.7) for estimating the dependence parameter $\theta$ in the second step; i.e. we work with $\mathbf{J}^{(\varepsilon)}(\mathbf{X}, \mathbf{Y} ; \eta)$ as given in Remark 3.4. As before we denote the resulting estimates by $\widetilde{\gamma}=(\log \widetilde{c}, \widetilde{\alpha})$ and $\widetilde{\eta}=(\log \widetilde{c}, \widetilde{\alpha}, \widetilde{\theta})$. Assume further that $\gamma_{0}=\left(\log c_{0}, \alpha_{0}\right)$ and $\eta_{0}=\left(\log c_{0}, \alpha_{0}, \theta_{0}\right)$ are the true parameter vectors.

Taylor expansions of each of the score functions in (3.6) and (3.8) separately yield

$$
\begin{aligned}
\left.\frac{\partial l_{12}^{(\varepsilon)}(\log c, \alpha)}{\partial \log c}\right|_{\gamma=\widetilde{\gamma}}= & \left.\frac{\partial l_{12}^{(\varepsilon)}(\gamma)}{\partial \log c}\right|_{\gamma=\gamma_{0}}+\left.\left(\log \widetilde{c}-\log c_{0}\right) \frac{\partial^{2} l_{12}^{(\varepsilon)}(\gamma)}{\partial(\log c)^{2}}\right|_{\gamma=\gamma_{* * *}}+\left.\left(\widetilde{\alpha}-\alpha_{0}\right) \frac{\partial^{2} l_{12}^{(\varepsilon)}(\gamma)}{\partial \alpha \partial \log c}\right|_{\gamma=\gamma_{* * *}} \\
\left.\frac{\partial l_{12}^{(\varepsilon)}(\log c, \alpha)}{\partial \alpha}\right|_{\gamma=\widetilde{\gamma}}= & \left.\frac{\partial l_{12}^{(\varepsilon)}(\gamma)}{\partial \alpha}\right|_{\gamma=\gamma_{0}}+\left.\left(\log \widetilde{c}-\log c_{0}\right) \frac{\partial^{2} l_{12}^{(\varepsilon)}(\gamma)}{\partial \log c \partial \alpha}\right|_{\gamma=\gamma_{* *}}+\left.\left(\widetilde{\alpha}-\alpha_{0}\right) \frac{\partial^{2} l_{12}^{(\varepsilon)}(\gamma)}{\partial \alpha^{2}}\right|_{\gamma=\gamma_{* *}} \\
\left.\frac{\partial l^{(\varepsilon)}(\log c, \alpha, \theta)}{\partial \theta}\right|_{\eta=\widetilde{\eta}}= & \left.\frac{\partial l^{(\varepsilon)}(\eta)}{\partial \theta}\right|_{\eta=\eta_{0}}+\left.\left(\log \widetilde{c}-\log c_{0}\right) \frac{\partial^{2} l^{(\varepsilon)}(\eta)}{\partial \log c \partial \theta}\right|_{\eta=\eta_{*}}+\left.\left(\widetilde{\alpha}-\alpha_{0}\right) \frac{\partial^{2} l^{(\varepsilon)}(\eta)}{\partial \alpha \partial \theta}\right|_{\eta=\eta_{*}} \\
& +\left.\left(\widetilde{\theta}-\theta_{0}\right) \frac{\partial^{2} l^{(\varepsilon)}(\eta)}{\partial \theta^{2}}\right|_{\eta=\eta_{*}}
\end{aligned}
$$

where $\gamma_{* * *}$ and $\gamma_{* *}$ are between $\widetilde{\gamma}=(\log \widetilde{c}, \widetilde{\alpha})$ and $\gamma_{0}=\left(\log c_{0}, \alpha_{0}\right)$, and $\eta_{*}$ is between $\widetilde{\eta}$ and $\eta_{0}$, componentwise.

Since the left hand sides of the equations in (4.12) are zero, so are the equations on the right hand side. Recall from Lemma 4.2 the matrix $H^{(\varepsilon)}=H^{(\varepsilon)}(\eta)$ of the second-order derivatives of the log-likelihoods and denote by $H_{*}^{(\varepsilon)}$ the matrix $H^{(\varepsilon)}$ at $\gamma_{* * *}, \gamma_{* *}$ and $\eta_{*}$ row-wise. Recalling the vector of score functions $\mathbf{J}^{(\varepsilon)}(\eta)$ from Remark 3.4, we rewrite (4.12) as

$$
H_{*}^{(\varepsilon)}\left(\widetilde{\eta}-\eta_{0}\right)=-\left.\mathbf{J}^{(\varepsilon)}(\eta)\right|_{\eta=\eta_{0}} .
$$


Rewrite the components of the vector $\mathbf{J}^{(\varepsilon)}(\eta)$ of the score functions in (3.6) and (3.8) as

$$
\begin{aligned}
\frac{\partial l_{12}^{(\varepsilon)}(\log c, \alpha)}{\partial \log c} & =2 \lambda^{(\varepsilon)} t\left(\frac{\widehat{\lambda}^{(\varepsilon)}}{\lambda^{(\varepsilon)}}-1\right) \\
\frac{\partial l_{12}^{(\varepsilon)}(\log c, \alpha)}{\partial \alpha} & =2 \lambda^{(\varepsilon)} t \log \varepsilon\left(\frac{\widehat{\lambda}^{(\varepsilon)}}{\lambda^{(\varepsilon)}}-1\right)-\sum_{i=1}^{n}\left(\log \left(\frac{Z_{i}}{\varepsilon}\right)-\frac{1}{\alpha}\right) \\
\frac{\partial l^{(\varepsilon)}(\log c, \alpha, \theta)}{\partial \theta} & =\sum_{i=1}^{n \|} T_{i}+\frac{n^{\|}}{\alpha+\theta}-\lambda^{(\varepsilon) \|_{t}} t \frac{\alpha \log 2}{\theta^{2}} \\
& =\sum_{i=1}^{n \|}\left(T_{i}-\mu_{T}\right)+\frac{\alpha \log 2}{\theta^{2}} \lambda^{(\varepsilon) \|_{t}} t\left(\frac{\widehat{\lambda}^{(\varepsilon) \|}}{\lambda^{(\varepsilon) \|}}-1\right) .
\end{aligned}
$$

The next result shows the consistency of the estimator $\widetilde{\eta}$.

Proposition 4.7. Assume the bivariate $\alpha$-stable Clayton subordinator with common marginal parameters $(\log c, \alpha)$ and dependence parameter $\theta=\alpha \delta$. Assume also the observation scheme as described in Section Q Let $b(\alpha, \theta)$ be defined as in (4.7) and assume that $b(\alpha, \theta) \neq 0$. Then the two-step estimator $\widetilde{\eta}$ is consistent; i.e., as $n^{\|} \rightarrow \infty$ (then also $n \rightarrow \infty$ ) for fixed $\varepsilon>0$,

$$
\widetilde{\eta} \stackrel{P}{\rightarrow} \eta .
$$

Proof. We denote again by $\eta_{0}$ the true parameter vector. Now divide (4.13) by $n$ and obtain

$$
\frac{1}{n} H_{*}^{(\varepsilon)}\left(\widetilde{\eta}-\eta_{0}\right)=-\left.\frac{1}{n} \mathbf{J}^{(\varepsilon)}(\eta)\right|_{\eta=\eta_{0}} .
$$

From the equations in (4.14), (4.15) and (4.16) the vector on the right hand side of (4.17) is given by

$$
\left.\frac{1}{n} \mathbf{J}^{(\varepsilon)}(\eta)\right|_{\eta=\eta_{0}}=\frac{2 \lambda^{(\varepsilon)} t}{n}\left(\begin{array}{c}
\frac{\widehat{\lambda}^{(\varepsilon)}}{\lambda^{(\varepsilon)}}-1 \\
\log \varepsilon\left(\frac{\widehat{\lambda}^{(\varepsilon)}}{\lambda^{(\varepsilon)}}-1\right)-\frac{1}{2 \lambda^{(\varepsilon)} t} \sum_{i=1}^{n}\left(\log \left(\frac{Z_{i}}{\varepsilon}\right)-\frac{1}{\alpha}\right) \\
\frac{1}{2 \lambda^{(\varepsilon)} t} \sum_{i=1}^{n \|}\left(T_{i}-\mu_{T}\right)+\frac{\alpha \log 2}{\theta^{2}} \frac{\lambda^{(\varepsilon) \|}}{2 \lambda^{(\varepsilon)}}\left(\frac{\widehat{\lambda}^{\varepsilon) \|}}{\lambda^{(\varepsilon) \|}}-1\right)
\end{array}\right)_{\eta=\eta_{0}} .
$$

Now, by the Marcinkiewicz-Zygmund SLLN (cf. e.g. [5], Theorem 2.5.10) $\widehat{\lambda}^{(\varepsilon)}=\frac{n}{2 t} \stackrel{\text { a.s. }}{\rightarrow} \lambda_{0}^{(\varepsilon)}$ as $n \rightarrow \infty$. Invoking the same argument for $\widehat{\lambda}^{(\varepsilon) \|}$ ensures that the second summand of the third component tends to 0 a.s. as $n^{\|} \rightarrow \infty$. For the terms involving $Z_{i}$ and $T_{i}$ we apply the SLLN for random sums (cf. e.g. [5], Lemma 2.5.3) and obtain that the right hand side of (4.17) tends to the zero vector a.s. as $n^{\|} \rightarrow \infty$ (and $\left.n \rightarrow \infty\right)$.

Next we show that the limit of $\frac{1}{n} H^{(\varepsilon)}\left(\eta_{0}\right)$ exists and is deterministic and independent of $t$ as $n^{\|} \rightarrow \infty$. Note that by the SLLN for random sums $A\left(\alpha_{0}, \theta_{0}\right)$ and $B\left(\alpha_{0}, \theta_{0}\right)$ converge a.s. to $a\left(\alpha_{0}, \theta_{0}\right)$ and $b\left(\alpha_{0}, \theta_{0}\right)$ as defined in (4.6) and (4.7), respectively. Using also the fact that $2 \lambda_{0}^{(\varepsilon)} t / n \stackrel{\text { a.s. }}{\rightarrow} 1$, we obtain $\frac{1}{n} H^{(\varepsilon)}\left(\eta_{0}\right) \stackrel{\text { a.s. }}{\rightarrow}-D$, where $D$ is given in (4.5) and is invertible by Remark 4.4. 
Now consider $H_{*}^{(\varepsilon)}$ and write $\lambda_{* * *}^{(\varepsilon)}=\left.\lambda^{(\varepsilon)}\right|_{\gamma_{* * *}}, \lambda_{* *}^{(\varepsilon)}=\left.\lambda^{(\varepsilon)}\right|_{\gamma_{* *}}, \lambda_{*}^{(\varepsilon)}=\left.\lambda^{(\varepsilon)}\right|_{\eta_{*}}, \alpha_{* *}=\left.\alpha\right|_{\gamma_{* *}}$, $\alpha_{*}=\left.\alpha\right|_{\eta_{*}} A_{*}=\left.A\right|_{\eta_{*}}, B_{*}=\left.B\right|_{\eta_{*}}$, and $d_{*}=\left.d\right|_{\eta_{*}}$. Then we calculate

$$
\operatorname{det} H_{*}^{(\varepsilon)}=-4 \frac{d_{*} B_{*}}{\alpha_{* *}^{2}} \lambda_{* * *}^{(\varepsilon)} \lambda_{*}^{(\varepsilon)} t^{2} n \neq 0
$$

for $\eta$ close to $\eta_{0}$ by continuity. Hence, $H_{*}^{(\varepsilon)}$ is invertible. From (4.17) we obtain

$$
\left(\frac{\log \widetilde{c}-\log c_{0}}{\log \varepsilon}, \widetilde{\alpha}-\alpha_{0}, \widetilde{\theta}-\theta_{0}\right)^{\top}=-\left(\frac{1}{n} H_{*}^{(\varepsilon)}\right)^{-1} \times\left.\frac{1}{n} \mathbf{J}^{(\varepsilon)}(\eta)\right|_{\eta=\eta_{0}},
$$

where

$$
\frac{1}{n} H_{*}^{(\varepsilon)}=\left(\begin{array}{ccc}
\frac{\lambda_{* * *}^{(\varepsilon)}}{\widehat{\lambda}^{(\varepsilon)}} & 0 & 0 \\
0 & \frac{\lambda_{* *}^{(\varepsilon)}}{\widehat{\lambda^{(}(\varepsilon)}} & 0 \\
0 & 0 & \frac{\lambda_{*}^{(\varepsilon)}}{\hat{\lambda}^{(\varepsilon)}}
\end{array}\right) \times\left(\begin{array}{ccc}
-\log \varepsilon & \log \varepsilon & 0 \\
(\log \varepsilon)^{2} & -\frac{\widehat{\lambda}^{(\varepsilon)}}{\alpha_{* *}^{2} \lambda_{* *}^{(\varepsilon)}}-(\log \varepsilon)^{2} & 0 \\
-d_{*} \frac{\alpha_{*} \log 2}{\theta_{*}^{2}} \log \varepsilon & d_{*}\left(\frac{\alpha_{*} \log 2}{\theta_{*}^{2}} \log \varepsilon-A_{*}\right) & -d_{*} B_{*}
\end{array}\right) .
$$

This implies that its inverse is given by

$$
\left(\frac{1}{n} H_{*}^{(\varepsilon)}\right)^{-1}=\left(\begin{array}{ccc}
-\frac{1}{\log \varepsilon}-\frac{\lambda_{* *}^{(\varepsilon)}}{\widehat{\lambda}^{(\varepsilon)}} \alpha_{* *}^{2} \log \varepsilon & -\frac{\lambda_{* *}^{(\varepsilon)}}{\widehat{\lambda}^{(\varepsilon)}} \alpha_{* *}^{2} & 0 \\
-\frac{\lambda_{* *}^{(\varepsilon)}}{\widehat{\lambda}^{(\varepsilon)}} \alpha_{* *}^{2} \log \varepsilon & -\frac{\lambda_{* *}^{(\varepsilon)}}{\widehat{\lambda}^{(\varepsilon)}} \alpha_{* *}^{2} & 0 \\
\frac{A_{*} \alpha_{* *}^{2}(\varepsilon)}{B_{*}^{(\varepsilon)}} \frac{\lambda_{* *}}{\hat{\lambda}_{(\varepsilon)}} \log \varepsilon+\frac{\alpha_{*} \log 2}{\theta_{*}^{2} B_{*}} & \frac{A_{*} \alpha_{* *}^{2}}{B_{*}} \frac{\lambda_{* *}^{(\varepsilon)}}{\hat{\lambda}^{(\varepsilon)}} & -\frac{1}{d_{*} B_{*}}
\end{array}\right) \times\left(\begin{array}{ccc}
\frac{\widehat{\lambda}^{(\varepsilon)}}{\lambda_{* * *}^{(\varepsilon)}} & 0 & 0 \\
0 & \frac{\widehat{\lambda}^{(\varepsilon)}}{\lambda_{* *}^{(\varepsilon)}} & 0 \\
0 & 0 & \frac{\widehat{\lambda}^{(\varepsilon)}}{\lambda_{*}^{(\varepsilon)}}
\end{array}\right) .
$$

All matrix elements are finite random variables and they remain bounded in probability for $n^{\|} \rightarrow \infty$, since $\widehat{\lambda}^{(\varepsilon)} \stackrel{\text { a.s. }}{\rightarrow} \lambda_{0}^{(\varepsilon)}$ and all starred values are between the estimates and the true parameter values. Hence we conclude that $\widetilde{\eta} \stackrel{P}{\rightarrow} \eta_{0}$.

We are now ready to formulate the main result of our paper.

Theorem 4.8. Assume a bivariate $\alpha$-stable Clayton subordinator with common marginal parameters $(\log c, \alpha)$ and dependence parameter $\theta=\alpha \delta$. Assume also the observation scheme as described in Section 国. Let $a(\alpha, \theta)$ and $b(\alpha, \theta)$ be defined as in (4.6) and (4.7), respectively, and let $m$ be as in (4.11). If $b(\alpha, \theta) \neq 0$, then as $\varepsilon \rightarrow 0$,

$$
\sqrt{2 c \varepsilon^{-\alpha} t}\left(\begin{array}{c}
\frac{\log \widetilde{c}-\log c}{\log \varepsilon} \\
\widetilde{\alpha}-\alpha \\
\widetilde{\theta}-\theta
\end{array}\right) \stackrel{d}{\rightarrow} N_{3}(\mathbf{0}, V)
$$

where

$$
V=\left(\begin{array}{ccc}
\alpha^{2} & \alpha^{2} & -\frac{\alpha^{2}(a+m)}{b} \\
\alpha^{2} & \alpha^{2} & -\frac{\alpha^{2}(a+m)}{b} \\
-\frac{\alpha^{2}(a+m)}{b} & -\frac{\alpha^{2}(a+m)}{b} & \frac{1}{b d}-\frac{3 \alpha^{2}(\log 2)^{2}}{b^{2} \theta^{4}}+\frac{a \alpha^{2}(a+2 m)}{b^{2}}
\end{array}\right)
$$


Proof. We start with the left hand side of equation in (4.17). Multiplying both sides of (4.17) by $\sqrt{n}$ yields as in the proof of Proposition 4.7 for $n^{\|}$(hence $n$ ) sufficiently large by consistency that $H_{*}^{(\varepsilon)}$ is invertible and by (4.18)

$$
\sqrt{n}\left(\frac{\log \widetilde{c}-\log c_{0}}{\log \varepsilon}, \widetilde{\alpha}-\alpha_{0}, \widetilde{\theta}-\theta_{0}\right)^{\top}=-\left(\frac{1}{n} H_{*}^{(\varepsilon)}\right)^{-1} \times\left.\frac{1}{\sqrt{n}} \mathbf{J}^{(\varepsilon)}(\eta)\right|_{\eta=\eta_{0}}
$$

The vector $\frac{1}{\sqrt{n}} \mathbf{J}^{(\varepsilon)}(\eta)$ is asymptotically normal with mean zero and covariance matrix $M=$ $\frac{1}{2 \lambda^{(\varepsilon)} t} \mathbb{E}\left[\frac{\partial l^{(\varepsilon)}(\eta)}{\partial \eta} \frac{\partial l^{(\varepsilon)}(\eta)^{T}}{\partial \eta}\right]$ calculated in Lemma 4.6. Since $\gamma^{* * *}$ and $\gamma^{* *}$ are between $\widetilde{\gamma}$ and $\gamma_{0}$ and $\eta^{*}$ between $\widetilde{\eta}$ and $\eta_{0}$, from the consistency of the estimators in Proposition 4.7, $\left(H_{*}^{(\varepsilon)}\right)^{-1}$ converges a.s. by the SLLN and the consistency of the parameters to $D^{-1}$ as calculated in Remark 4.4. Consequently, $\sqrt{n}\left(\frac{\log \widetilde{c}-\log c_{0}}{\log \varepsilon}, \widetilde{\alpha}-\alpha_{0}, \widetilde{\theta}-\theta_{0}\right)^{\top}$ converges as $n \rightarrow \infty$ in distribution to a normal vector with asymptotic covariance matrix

$$
\begin{aligned}
G^{-1} & =\left.D^{-1} M\left(D^{-1}\right)^{\top}\right|_{\eta=\eta_{0}} \\
& =\left(\begin{array}{ccc}
\frac{1}{(\log \varepsilon)^{2}}+\alpha_{0}^{2} & \alpha_{0}^{2} & -\frac{\left(a_{0}+m_{0}\right) \alpha_{0}^{2}}{b_{0}}+\frac{\alpha_{0} \log 2}{b_{0} \theta_{0}^{2} \log \varepsilon} \\
\alpha_{0}^{2} & \alpha_{0}^{2} & -\frac{\left(a_{0}+m_{0}\right) \alpha_{0}^{2}}{b_{0}} \\
-\frac{\left(a_{0}+m_{0}\right) \alpha_{0}^{2}}{b_{0}}+\frac{\alpha_{0} \log 2}{b_{0} \theta_{0}^{2} \log \varepsilon} & -\frac{\left(a_{0}+m_{0}\right) \alpha_{0}^{2}}{b_{0}} & \frac{1}{b_{0} d_{0}}-\frac{3 \alpha_{0}^{2}(\log 2)^{2}}{b_{0}^{2} \theta_{0}^{4}}+\frac{a_{0} \alpha_{0}^{2}\left(a_{0}+2 m_{0}\right)}{b_{0}^{2}}
\end{array}\right)
\end{aligned}
$$

with $G$ as in (4.1).

Then by the SLLN we know that $\frac{\widehat{\lambda}^{((\varepsilon)}}{\lambda^{(\varepsilon)}}=\frac{n}{2 \lambda^{(\varepsilon)} t}=\frac{n}{2 c \varepsilon^{-\alpha} t} \stackrel{\text { a.s. }}{\rightarrow} 1$ as $n \rightarrow \infty$ (either $\varepsilon \downarrow 0$ or $t \rightarrow \infty)$, hence the rate $\sqrt{n}$ can be replaced by $\sqrt{2 c t \varepsilon^{-\alpha}}$. Finally, $G^{-1} \rightarrow V$ as $\varepsilon \rightarrow 0$ and this completes the proof.

Remark 4.9. (i) Note that for $t \rightarrow \infty$ and fixed $\varepsilon>0$ the asymptotic covariance matrix in (4.19) is given by $G^{-1}$.

(ii) The above theorem implies that the normal limit vector has representation

$$
\left(N_{1}, N_{1}, N_{2}\right)^{\top}
$$

where $N_{1}$ has variance $\alpha^{2}, N_{2}$ has variance $\frac{1}{b d}+\frac{\alpha^{2}}{b^{2}}\left(-\frac{3(\log 2)^{2}}{\theta^{4}}+a(a+2 m)\right)$, and the correlation between $N_{1}$ and $N_{2}$ is given by

$$
\operatorname{Corr}\left(N_{1}, N_{2}\right)=-\frac{a+m}{\sqrt{\frac{1}{\alpha^{2} d}-\frac{3(\log 2)^{2}}{b \theta^{4}}+\frac{a}{b}(a+2 m)}} .
$$

Example 4.10. [Asymptotic covariance matrix for a bivariate $\alpha$-stable Clayton subordinator] Let $\mathbf{S}=(\mathbf{S}(t))_{t \geq 0}$ be a bivariate $\alpha$-stable subordinator with a Clayton Lévy copula as introduced in Example 2.2. Assume further its parameters $c_{1}=c_{2}=c, \alpha_{1}=\alpha_{2}=\alpha$ and $\theta=\alpha \delta$ are estimated by a two-step method as in Section 3.2. The asymptotic covariance matrix as $\varepsilon \rightarrow 0$ for the model with parameter values $c=1, \alpha=0.5, \theta=1$ can be computed numerically similar 
to the calculation at the end of Section 5 in [7]. Note that it involves the numerical integration of certain integrals. We find the asymptotic covariance matrix of $\widetilde{\eta}=(\log \widetilde{c}, \widetilde{\alpha}, \widetilde{\theta})$ as

$$
V=\left[\begin{array}{ccc}
0.25 & 0.25 & 0.1042 \\
0.25 & 0.25 & 0.1042 \\
0.1042 & 0.1042 & 2.6273
\end{array}\right]
$$

Alternatively, this matrix can also be estimated replacing the numerical integration by a Monte Carlo simulation. For this the expectations in (4.6) and (4.7) and the covariance $m$ from (4.11) are empirically estimated by generating bivariate observations from (3.4) with parameter values mentioned above. Based on $10^{6}$ bivariate observations, this yields the same asymptotic covariance matrix as above (4 leading decimals coindice).

From this, we calculate $\operatorname{Corr}\left(N_{1}, N_{2}\right)=0.1286$.

Remark 4.11. For the bivariate $\alpha$-stable Clayton Lévy process with equal marginal processes we have been able to calculate the Godambe information matrix analytically. However, for most models this is too complicated. It requires derivatives of first and second order, the integration of some compound functions and the inverses and multiplications of possibly high dimensional matrices. As an alternative, a jackknife resampling method has been suggested and can also be applied in this context for arbitrary Lévy processs; cf. Joe [10], Section 10.1, and references given there.

\section{Maximum likelihood estimation of the full model}

We compare the two-step procedure presented in Section 2 with two alternatives. Firstly, we consider the estimation method presented in [7] based on only common jumps. Secondly, we also compare this method with the full likelihood, based on single and common jumps. For this reason we present here the likelihood function of the full model. The observation scheme is as explained in Section 2, where $n^{\|}+n_{1}^{\perp}+n_{2}^{\perp}$ is the number of observations.

From (2.6) the Lévy densities of $\Pi_{1}^{(\varepsilon) \perp}, \Pi_{2}^{(\varepsilon) \perp}$ and $\Pi^{(\varepsilon) \|}$ are given by

$$
\begin{aligned}
\nu_{1}^{\perp}(x) & =c \alpha x^{-\alpha-1}\left[1-\left(1+(x / \varepsilon)^{-\alpha \delta}\right)^{-1 / \delta-1}\right], \quad x>\varepsilon \\
\nu_{2}^{\perp}(y) & =c \alpha y^{-\alpha-1}\left[1-\left(1+(y / \varepsilon)^{-\alpha \delta}\right)^{-1 / \delta-1}\right], \quad y>\varepsilon, \\
\nu^{\|}(x, y) & =c \alpha^{2}(1+\delta)(x y)^{\alpha \delta-1}\left(x^{\alpha \delta}+y^{\alpha \delta}\right)^{-1 / \delta-2} .
\end{aligned}
$$

As intensities we obtain from (2.7) $\lambda^{(\varepsilon) \|}=c 2^{-1 / \delta} \varepsilon^{-\alpha}$. Moreover, the marginal intensities are $\lambda_{1}^{(\varepsilon)}=\lambda_{2}^{(\varepsilon)}=c \varepsilon^{-\alpha}$, so that $\lambda_{1}^{(\varepsilon) \perp}=\lambda_{2}^{(\varepsilon) \perp}=c \varepsilon^{-\alpha}\left(1-2^{-1 / \delta}\right)$. This implies the intensity of the bivariate $\operatorname{CPP} \rho^{(\varepsilon)}:=\lambda^{(\varepsilon) \|}+\lambda_{1}^{(\varepsilon) \perp}+\lambda_{2}^{(\varepsilon) \perp}=c \varepsilon^{-\alpha}\left(2-2^{-1 / \delta}\right)$.

For simplicity we reparameterize the model again as in Section 3.2 by setting $\alpha \delta=\theta$ and take $\log c$ instead of $c$ as second marginal parameter. Now we recall Th. 4.1 of [6] for a bivariate $\mathrm{CPP}$ and obtain the likelihood function; here $\left(x_{i}, y_{i}\right)_{i=1, \ldots, n} \|$ denote the common jumps in both 
components and $\widetilde{x}_{i}$ for $i=1, \ldots, n_{1}^{\perp}$ and $\widetilde{y}_{i}$ for $i=1, \ldots, n_{2}^{\perp}$ denote the single jumps. The likelihood function of the bivariate CPP is then given by

$$
\begin{aligned}
L^{(\varepsilon)}(\log c, \alpha, \theta)= & \left(e^{-\rho^{(\varepsilon)} t} \prod_{i=1}^{n^{\|}} \nu^{\|}\left(x_{i}, y_{i}\right)\right) \times\left(e^{-\lambda_{1}^{(\varepsilon) \perp} t} \prod_{i=1}^{n_{1}^{\perp}} \nu_{1}^{\perp}\left(\widetilde{x}_{i}\right)\right) \times\left(e^{-\lambda_{2}^{(\varepsilon) \perp} t} \prod_{i=1}^{n_{2}^{\perp}} \nu\left(\widetilde{y}_{i}\right)\right) \\
= & e^{-c t \varepsilon^{-\alpha}\left(2-2^{-\alpha / \theta}\right)}(\alpha+\theta)^{n \|}(\alpha c)^{n \|+n_{1}^{\perp}+n_{2}^{\perp}} \prod_{i=1}^{n^{\|}}\left[\left(x_{i} y_{i}\right)^{\theta-1}\left(x_{i}^{\theta}+y_{i}^{\theta}\right)^{-\alpha / \theta-2}\right] \\
& \times \prod_{i=1}^{n_{1}^{\perp}}\left[\widetilde{x}_{i}^{-\alpha-1}\left(1-\left(1+\left(\widetilde{x}_{i} / \varepsilon\right)^{-\theta}\right)^{-\alpha / \theta-1}\right)\right] \\
& \times \prod_{i=1}^{n_{2}^{\perp}}\left[\widetilde{y}_{i}^{-\alpha-1}\left(1-\left(1+\left(\widetilde{y}_{i} / \varepsilon\right)^{-\theta}\right)^{-\alpha / \theta-1}\right)\right]
\end{aligned}
$$

The log-likelihood is given by

$$
\begin{aligned}
l^{(\varepsilon)}(\log c, \alpha, \theta)= & -c t \varepsilon^{-\alpha}\left(2-2^{-\alpha / \theta}\right)+n^{\|} \log (\alpha+\theta)+\left(n^{\|}+n_{1}^{\perp}+n_{2}^{\perp}\right)(\log \alpha+\log c) \\
& +(\theta-1) \sum_{i=1}^{n^{\|}}\left(\log x_{i}+\log y_{i}\right)-\left(2+\frac{\alpha}{\theta}\right) \sum_{i=1}^{n^{\|}} \log \left(x_{i}^{\theta}+y_{i}^{\theta}\right) \\
& -(\alpha+1) \sum_{i=1}^{n_{1}^{\perp}} \log \widetilde{x}_{i}+\sum_{i=1}^{n_{1}^{\perp}} \log \left[1-\left(1+\left(\widetilde{x}_{i} / \varepsilon\right)^{-\theta}\right)^{-\alpha / \theta-1}\right] \\
& -(\alpha+1) \sum_{i=1}^{n_{2}^{\perp}} \log \widetilde{y}_{i}+\sum_{i=1}^{n_{2}^{\perp}} \log \left[1-\left(1+\left(\widetilde{y}_{i} / \varepsilon\right)^{-\theta}\right)^{-\alpha / \theta-1}\right] .
\end{aligned}
$$

For the score functions we obtain

$$
\begin{aligned}
\frac{\partial l^{(\varepsilon)}}{\partial \log c}= & -c t \varepsilon^{-\alpha}\left(2-2^{-\alpha / \theta}\right)+\frac{n^{\|}+n_{1}^{\perp}+n_{2}^{\perp}}{c} \\
\frac{\partial l^{(\varepsilon)}}{\partial \alpha}= & c t \varepsilon^{-\alpha}\left(2 \log \varepsilon-2^{-\alpha / \theta} \log \varepsilon-\frac{2^{-\alpha / \theta} \log 2}{\theta}\right)+\frac{n^{\|}}{\alpha+\theta}+\frac{n^{\|}+n_{1}^{\perp}+n_{2}^{\perp}}{\alpha} \\
& -\frac{1}{\theta} \sum_{i=1}^{n^{\|}} \log \left(x_{i}^{\theta}+y_{i}^{\theta}\right)-\sum_{i=1}^{n_{1}^{\perp}} \log \widetilde{x}_{i}+\sum_{i=1}^{n_{1}^{\perp}} \frac{\partial}{\partial \alpha} \log \left[1-\left(1+\left(\widetilde{x}_{i} / \varepsilon\right)^{-\theta}\right)^{-\alpha / \theta-1}\right] \\
& -\sum_{i=1}^{n_{2}^{\perp}} \log \widetilde{y}_{i}+\sum_{i=1}^{n_{2}^{\perp}} \frac{\partial}{\partial \alpha} \log \left[1-\left(1+\left(\widetilde{y}_{i} / \varepsilon\right)^{-\theta}\right)^{-\alpha / \theta-1}\right] \\
\frac{\partial l^{(\varepsilon)}}{\partial \theta}= & \frac{c t \alpha \varepsilon^{-\alpha} 2^{-\alpha / \theta} \log 2}{\theta^{2}}+\frac{n^{\|}}{\alpha+\theta}+\sum_{i=1}^{n^{\|}}\left(\log x_{i}+\log y_{i}\right)+\frac{\alpha}{\theta^{2}} \sum_{i=1}^{n^{\|}} \log \left(x_{i}^{\theta}+y_{i}^{\theta}\right) \\
& -\left(2+\frac{\alpha}{\theta}\right) \sum_{i=1}^{n^{\Perp}} \frac{\partial}{\partial \theta} \log \left(x_{i}^{\theta}+y_{i}^{\theta}\right)+\sum_{i=1}^{n_{1}} \frac{\partial}{\partial \theta} \log \left[1-\left(1+\left(\widetilde{x}_{i} / \varepsilon\right)^{-\theta}\right)^{-\alpha / \theta-1}\right] \\
& +\sum_{i=1}^{n_{2}^{\perp}} \frac{\partial}{\partial \theta} \log \left[1-\left(1+\left(\widetilde{y}_{i} / \varepsilon\right)^{-\theta}\right)^{-\alpha / \theta-1}\right] .
\end{aligned}
$$




\begin{tabular}{|c|c|c|c|c|c|}
\hline Method of estimation & Truncation point & & $c=1$ & $\alpha=0.5$ & $\delta=2$ \\
\hline \multirow{6}{*}{$\begin{array}{c}\text { MLE } \\
\text { (only bivariate jumps) } \\
\text { as in [7] }\end{array}$} & \multirow{3}{*}{$\varepsilon=0.001$} & Mean & 1.0678 & 0.5289 & 2.1489 \\
\hline & & $\sqrt{M S E}$ & 0.6344 & 0.1206 & 0.9511 \\
\hline & & $M R B$ & 0.0517 & 0.0525 & 0.0842 \\
\hline & \multirow{3}{*}{$\varepsilon=0.00001$} & Mean & 1.0460 & 0.5020 & 2.0301 \\
\hline & & $\sqrt{M S E}$ & 0.3677 & 0.0349 & 0.2488 \\
\hline & & $M R B$ & 0.0413 & 0.0044 & 0.0144 \\
\hline \multirow{6}{*}{$\begin{array}{c}\text { MLE } \\
\text { (full model) } \\
\text { as in Section } 3\end{array}$} & \multirow{3}{*}{$\varepsilon=0.001$} & Mean & 1.0177 & 0.5216 & 2.0129 \\
\hline & & $\sqrt{M S E}$ & 0.5248 & 0.0777 & 0.4337 \\
\hline & & $M R B$ & 0.0072 & 0.0423 & 0.0119 \\
\hline & \multirow{3}{*}{$\varepsilon=0.00001$} & Mean & 1.0175 & 0.5021 & 2.0091 \\
\hline & & $\sqrt{M S E}$ & 0.2808 & 0.0239 & 0.1253 \\
\hline & & $M R B$ & 0.0142 & 0.0045 & 0.0042 \\
\hline \multirow{6}{*}{$\begin{array}{c}\text { IFM } \\
\text { (two-step method) } \\
\text { as in Section } 3.2\end{array}$} & \multirow{3}{*}{$\varepsilon=0.001$} & Mean & 1.0453 & 0.5231 & 2.0762 \\
\hline & & $\sqrt{M S E}$ & 0.5535 & 0.0859 & 0.6764 \\
\hline & & $M R B$ & 0.0264 & 0.0471 & 0.0379 \\
\hline & \multirow{3}{*}{$\varepsilon=0.00001$} & Mean & 1.0301 & 0.5021 & 2.0149 \\
\hline & & $\sqrt{M S E}$ & 0.3003 & 0.0257 & 0.1696 \\
\hline & & $M R B$ & 0.0249 & 0.0048 & 0.0065 \\
\hline
\end{tabular}

Table 6.1: Comparison of estimates for a bivariate $\frac{1}{2}$-stable Clayton process with common marginal parameters. We simulated 100 sample paths and estimated all parameters 100 times. Each of the 100 estimates was based on one sample path, on which all three methods were performed. From each sample path we truncated the small jumps based on the two truncation points $(\varepsilon=0.001$ and $\varepsilon=0.00001)$, respectively. Each sample path of the process was simulated as a continuous time realization of a $\mathrm{CPP}$ in one unit of time, $0 \leq t<1$, for $\tau=1000$, equivalent to truncation of the small jumps at the cut-off point $\xi=\bar{\Pi}^{\leftarrow}(\tau)=10^{-6}$.

The three parameters are obtained by numerical optimization.

It is possible to prove joint asymptotic normality of $(\log c, \alpha, \theta)$ similar to our calculations in Esmaeili and Klüppelberg [7] and in Section 3 of the present paper. However, for the observation scheme of the present paper this is even more complicated than in [7. We refrain from this tedious analytic exercise and, instead, present the results of a simulation study in the next section, where we compare all three methods presented.

\section{Comparison of estimation procedures}

In this section we compare the quality of the MLEs $\widehat{\eta}=(\log \widehat{c}, \widehat{\alpha}, \widehat{\theta})$ of the full model of Section 5 with the estimates $\widetilde{\eta}=(\log \widetilde{c}, \widetilde{\alpha}, \widetilde{\theta})$ obtained by the two-step method in Section 4 . Moreover, we also include in our comparison those estimates obtained from bivariate jumps larger than $\varepsilon$ only as derived in Th. 4.6 of [7]. Since this last method means to base the statistical analysis on less data, we expect that this method is less efficient than the MLE based on all available data. More 
precisely, for the first two parameters $\log c$ and $\alpha$, the rate has simply changed from $\sqrt{c 2^{-\alpha / \theta} \varepsilon^{-\alpha} t}$ to $\sqrt{c 2 \varepsilon^{-\alpha} t}$.

\section{The simulation study}

We simulate sample paths of the bivariate $\alpha$-stable Clayton subordinator with equal marginals and parameters given by $c=1(\log c=0), \alpha=1 / 2$ and $\delta=2(\theta=1)$. We generate sample paths of this process over a time span $[0, t]$, where we choose $t=1$ for simplicity. Recall from our observation scheme introduced in Section 2 that we observe all jumps larger than $\varepsilon$ either in one component or in both. Obviously, we cannot simulate a trajectory of a stable process, since we are restricted to the simulation of a finite number of jumps. For simulation purposes we choose a threshold $\xi$ (which should be much smaller than $\varepsilon$ ) and simulate jumps larger than $\xi$ in one component, and arbitrary in the second component. To this end we invoke Algorithm 6.15 in Cont and Tankov [3].

The simulation of a bivariate $\alpha$-stable Clayton subordinator is explained in detail in Example 6.18 of [3]. The algorithm starts by fixing a number $\tau$ determined by the required precision. This number coincides with the jump intensity $\lambda_{1}^{(\xi)}$, which fixes the average number of terms in the approximating CPP. More details can be found in [7.

For the estimation we first consider $\varepsilon=0.001$, i.e. a relatively large truncation point. Not surprisingly, the MLEs based on the full model discussed in Section 5 are definitely better than the other estimates in Table 6.1. We find it, however, remarkable that the two-step method outperforms the MLE based on joint jumps only. The reason for this is presumably that the MLE's based only on joint jumps use only such data with Lévy measure on $[\varepsilon, \infty)^{2}$. The two-step method, however, uses also data, which are only in one component larger than $\varepsilon$ in its first step. The marginal parameters are based on substantially more data.

When we consider also smaller jumps; i.e., if we choose $\varepsilon=10^{-5}$, the estimates will be more precise with less variation and smaller bias. In Table 6.1, the results in the lower part of each estimation method show this fact. It can also be seen from this table that the MLEs from a full model have the least mean relative bias (MRB) and mean square errors (MSE) as expected. In Figure 2 we visualize the situation for the this jump truncation point of $\varepsilon=10^{-5}$ based on 1000 simulated sample paths. Again all three estimation methods are performed for each sample path.

\section{Acknowledgement}

Both authors are grateful to Alexander Lindner for pointing out some inconsistencies in the proof or Proposition 4.7. His remarks lead also to an improvement of the presentation of our results. C.K. gratefully acknowledges financial support from the Institute for Advanced Study of the Technische Universität München (TUM-IAS). 
$\log c=0$
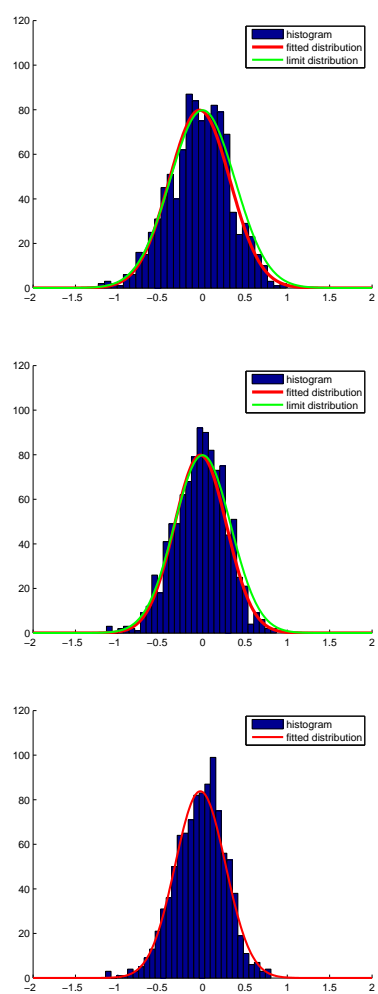

$\alpha=0.5$
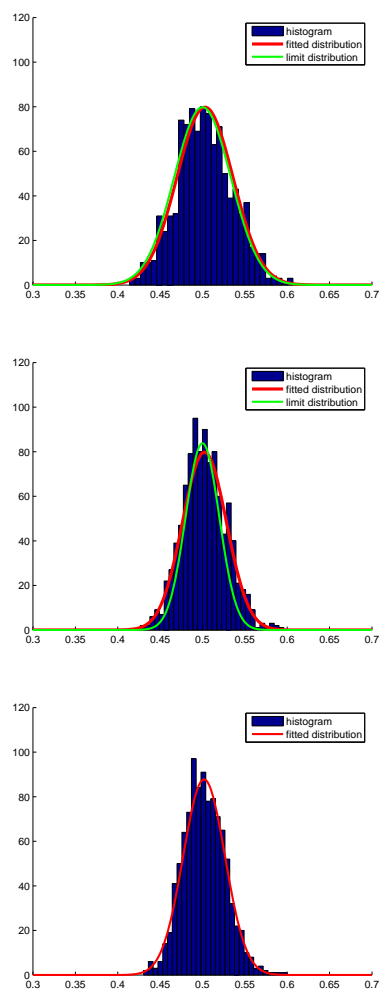

$\theta=1$
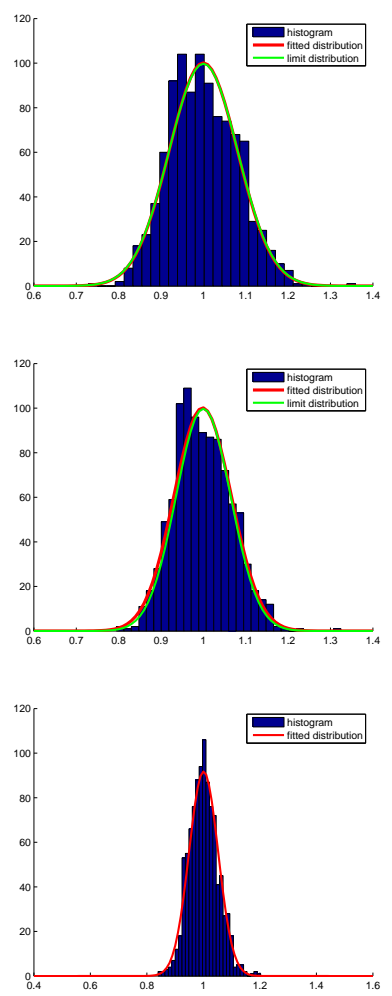

Figure 2: Histogram with statistically fitted normal density (red) and theoretical limit distribution (green) for 1000 parameter estimates of a bivariate Clayton stable Lévy process. The parameter values are $c=1, \alpha=0.5$ and $\delta=2$ and the jump-truncated point is $\varepsilon=0.00001$. The estimation procedures are MLEs based on joint jumps only (first row, limit distribution derived in Theorem 4.6 of [7]), the two-step method (second row, limit distribution derived in Theorem 4.8 above) and MLEs based on all jumps (third row, without theoretical limit law).

\section{References}

[1] I.V. Basawa and P.J. Brockwell. Inference for gamma and stable processes. Biometrika, 65(1):129-133, 1978.

[2] I.V. Basawa and P.J. Brockwell. A note on estimation for gamma and stable processes. Biometrika, 67(1):234-236, 1980.

[3] R. Cont and P. Tankov. Financial Modelling with Jump Processes. Chapman \& Hall, Boca Raton, 2004.

[4] I. Eder and C. Klüppelberg. Pareto Lévy measures and multivariate regular variation. Adv. in Appl. Probab., 44(1):117-138, 2012.

[5] P. Embrechts, C. Klüppelberg and T. Mikosch. Modelling Extremal Events for Insurance and Finance. Springer, Heidelberg, 1997. 
[6] H. Esmaeili and C. Klüppelberg. Parameter estimation of a bivariate compound poisson process. Insurance: Mathematics and Economics, 47:224-233, 2010.

[7] H. Esmaeili and C. Klüppelberg. Parameter estimation of a bivariate stable Lévy process. J. Mult. Anal., 102(5):918-930, 2011.

[8] V.P. Godambe. Estimating Functions. Oxford University Press, Oxford, 1991.

[9] R. Höpfner and J. Jacod. Some remarks on the joint estimation of the index and the scale parameter for stable processes. In: P. Mandl and M. Huskova (Eds.) Asymptotic Statistics. Proceedings of the Fifth Prague Symposium 1993, pp. 273-284. Physica Verlag, Heidelberg, 1994.

[10] H. Joe. Multivariate Models and Dependence Concepts. Chapman \& Hall/CRC, London, 1997.

[11] J. Kallsen and P. Tankov. Characterization of dependence of multidimensional Lévy processes using Lévy copulas. J. Mult. Anal., 97:1551-1572, 2006.

[12] S. I. Resnick. Extreme Values, Regular Variation, and Point Processes. Springer, New York, 1987.

[13] K. Sato. Lévy Processes and Infinitely Divisible Distributions. Cambridge University Press, Cambridge, U.K., 1999.

[14] Van der Vaart, A. W. Asymptotic Statistics. Cambridge University Press, Cambridge, 2007.

[15] J.J. Xu. Statistical Modelling and Inference for Multivariate and Longitudinal Discrete Response Data. Ph.D. Thesis, University of British Columbia, Department of Statistics, 1996.

\section{Appendix}

Proof of Lemma 4.2. The score functions in (3.6) have derivatives

$$
\begin{aligned}
& \frac{\partial^{2} l_{12}^{(\varepsilon)}(\log c, \alpha)}{\partial(\log c)^{2}}=-2 c t \varepsilon^{-\alpha}=-2 \lambda^{(\varepsilon)} t \\
& \frac{\partial^{2} l_{12}^{(\varepsilon)}(\log c, \alpha)}{\partial \alpha \partial \log c}=\frac{\partial^{2} l_{12}^{(\varepsilon)}(\log c, \alpha)}{\partial \log c \partial \alpha}=2 c t \varepsilon^{-\alpha} \log \varepsilon=2 \lambda^{(\varepsilon)} t \log \varepsilon \\
& \frac{\partial^{2} l_{12}^{(\varepsilon)}(\log c, \alpha)}{\partial \alpha^{2}}=-\frac{n}{\alpha^{2}}-2 c t \varepsilon^{-\alpha}(\log \varepsilon)^{2}=-\frac{n}{\alpha^{2}}-2 \lambda^{(\varepsilon)} t(\log \varepsilon)^{2} .
\end{aligned}
$$

This means that the upper left $2 \times 2$-matrix of $H^{(\varepsilon)}$ is the Fisher information matrix to the MLE of $(\log c, \alpha)$, calculated by Basawa and Brockwell [2], and also presented in Esmaeili and Klüppelberg [7, Example 3.1 (up to a deterministic factor), since here all observations from both 
marginals are considered. Since the score functions in (3.6) are independent of the parameter $\theta$, the matrix $H^{(\varepsilon)}$ has the structure as given in (4.5). It remains to calculate the last row of $H^{(\varepsilon)}$. We calculate the derivatives of the score function in (3.8) as follows: first, by Lemma 4.1.

$$
\frac{\partial^{2} l^{(\varepsilon)}(\log c, \alpha, \theta)}{\partial \log c \partial \theta}=-\frac{\partial^{2} \lambda^{(\varepsilon) \|}}{\partial \log c \partial \theta} t=-\lambda^{(\varepsilon) \|_{t}} \frac{\alpha \log 2}{\theta^{2}} .
$$

Furthermore,

$$
\begin{aligned}
\frac{\partial^{2} l^{(\varepsilon)}(\log c, \alpha, \theta)}{\partial \alpha \partial \theta}= & -\frac{\partial^{2} \lambda^{(\varepsilon) \|}}{\partial \alpha \partial \theta} t-\frac{n^{\|}}{(\alpha+\theta)^{2}}+\frac{1}{\theta^{2}} \sum_{i=1}^{n^{\|}} \log \left(X_{i}^{\theta}+Y_{i}^{\theta}\right) \\
& -\frac{1}{\theta} \sum_{i=1}^{n^{\|}} \frac{\partial}{\partial \theta} \log \left(X_{i}^{\theta}+Y_{i}^{\theta}\right) \\
\frac{\partial^{2} l^{(\varepsilon)}(\log c, \alpha, \theta)}{\partial \theta^{2}}= & -\frac{\partial^{2} \lambda^{(\varepsilon) \|}}{\partial \theta^{2}} t-\frac{n^{\|}}{(\alpha+\theta)^{2}}-\frac{2 \alpha}{\theta^{3}} \sum_{i=1}^{n^{\|}} \log \left(X_{i}^{\theta}+Y_{i}^{\theta}\right) \\
& +\frac{2 \alpha}{\theta^{2}} \sum_{i=1}^{n^{\|}} \frac{\partial}{\partial \theta} \log \left(X_{i}^{\theta}+Y_{i}^{\theta}\right)-\left(2+\frac{\alpha}{\theta}\right) \sum_{i=1}^{n^{\|}} \frac{\partial^{2}}{\theta^{2}} \log \left(X_{i}^{\theta}+Y_{i}^{\theta}\right) .
\end{aligned}
$$

Proof of Lemma 4.3. Since $D=-\frac{1}{2 \lambda^{(\varepsilon)} t} \mathbb{E}\left[H^{(\varepsilon)}\right]$, it remains to calculate the expectations:

$$
\begin{aligned}
\mathbb{E}[A(\alpha, \theta)]= & -\frac{\alpha(\log 2)^{2}}{\theta^{3}}+\frac{\log 2}{\theta^{2}}+\frac{1}{(\alpha+\theta)^{2}}-\frac{1}{\theta^{2}} \mathbb{E}\left[\log \left(X_{1}^{\theta}+Y_{1}^{\theta}\right)\right] \\
& +\frac{1}{\theta} \mathbb{E}\left[\frac{\partial}{\partial \theta} \log \left(X_{1}^{\theta}+Y_{1}^{\theta}\right)\right] \\
= & a(\alpha, \theta) \\
\mathbb{E}[B(\alpha, \theta)]= & \left(\frac{\alpha \log 2}{\theta^{2}}\right)^{2}-\frac{2 \alpha \log 2}{\theta^{3}}+\frac{1}{(\alpha+\theta)^{2}}+\frac{2 \alpha}{\theta^{3}} \mathbb{E}\left[\log \left(X_{1}^{\theta}+Y_{1}^{\theta}\right)\right] \\
& -\frac{2 \alpha}{\theta^{2}} \mathbb{E}\left[\frac{\partial}{\partial \theta} \log \left(X_{1}^{\theta}+Y_{1}^{\theta}\right)\right]+\left(2+\frac{\alpha}{\theta}\right) \mathbb{E}\left[\frac{\partial^{2}}{\partial \theta^{2}} \log \left(X_{1}^{\theta}+Y_{1}^{\theta}\right)\right] \\
= & b(\alpha, \theta) .
\end{aligned}
$$

Proof of Lemma 4.6. Recall the definition of the $Z_{i}$ for $i=1, \ldots, n$ as in Step 1 of Section 3.2 and the fact that $\log \frac{Z_{1}}{\varepsilon}, \ldots, \log \frac{Z_{n}}{\varepsilon}$ are exponential random variables with expectation $\alpha^{-1}$, and that in this first step they are treated as independent. The entries of the matrix $M=\left(m_{i j}\right)_{1 \leq i, j \leq 3}$ are calculated from the score functions (3.9) in Remark 3.4 as follows:

$$
\begin{aligned}
& m_{11}=\frac{1}{2 \lambda^{(\varepsilon)} t} \mathbb{E}\left[\left(\frac{\partial l_{12}^{(\varepsilon)}}{\partial \log c}\right)^{2}\right]=\frac{1}{2 \lambda^{(\varepsilon)} t} \mathbb{E}\left[-\frac{\partial^{2} l_{12}^{(\varepsilon)}}{\partial(\log c)^{2}}\right]=d_{11} \\
& m_{12}=\frac{1}{2 \lambda^{(\varepsilon)} t} \mathbb{E}\left[\left(\frac{\partial l_{12}^{(\varepsilon)}}{\partial \alpha}\right)\left(\frac{\partial l_{12}^{(\varepsilon)}}{\partial \log c}\right)\right]=\frac{1}{2 \lambda^{(\varepsilon)} t} \mathbb{E}\left[-\frac{\partial^{2} l_{12}^{(\varepsilon)}}{\partial \alpha \partial \log c}\right]=d_{12}=d_{21}=m_{21} \\
& m_{22}=\frac{1}{2 \lambda^{(\varepsilon)} t} \mathbb{E}\left[\left(\frac{\partial l_{12}^{(\varepsilon)}}{\partial \alpha}\right)^{2}\right]=\frac{1}{2 \lambda^{(\varepsilon)} t} \mathbb{E}\left[-\frac{\partial^{2} l_{12}^{(\varepsilon)}}{\partial \alpha^{2}}\right]=d_{22} .
\end{aligned}
$$


We abbreviate $T_{i}:=T\left(X_{i}, Y_{i}\right)$ and find from Lemma 4.4 in [7] that $\mu_{T}:=\mathbb{E}\left(T_{i}\right)=\frac{\alpha \log 2}{\theta^{2}}-\frac{1}{\alpha+\theta}$. Then by (3.6) and (3.8) we find

$$
\begin{aligned}
m_{13} & =\frac{1}{2 \lambda^{(\varepsilon)} t} \mathbb{E}\left[\left(\frac{\partial l_{12}^{(\varepsilon)}}{\partial \log c}\right)\left(\frac{\partial l^{(\varepsilon)}}{\partial \theta}\right)\right] \\
& =\frac{1}{2 \lambda^{(\varepsilon)} t} \mathbb{E}\left[\left(n-2 \lambda^{(\varepsilon)} t\right)\left(-\lambda^{(\varepsilon){ }_{t}} \frac{\alpha \log 2}{\theta^{2}}+\frac{n^{\|}}{\alpha+\theta}+\sum_{i=1}^{n^{\|}}\left(T_{i}-\mu_{T}\right)+n^{\|}\left(\frac{\alpha \log 2}{\theta^{2}}-\frac{1}{\alpha+\theta}\right)\right)\right] \\
& =\frac{1}{2 \lambda^{(\varepsilon)} t} \mathbb{E}\left[\left(n-2 \lambda^{(\varepsilon)} t\right)\left(\frac{\alpha \log 2}{\theta^{2}}\left(n^{\|}-\lambda^{(\varepsilon) \|} t\right)+\sum_{i=1}^{n^{\|}}\left(T_{i}-\mu_{T}\right)\right)\right] \\
& =\frac{\alpha \log 2}{2 \lambda^{(\varepsilon)} t \theta^{2}} \mathbb{E}\left[n\left(n^{\|}-\lambda^{(\varepsilon)} \|_{t}\right)\right]+\frac{1}{2 \lambda^{(\varepsilon)} t} \mathbb{E}\left[\mathbb{E}\left[n \sum_{i=1}^{n^{\|}}\left(T_{i}-\mu_{T}\right) \mid n, n^{\|}\right]\right] \\
& =\frac{\alpha \log 2}{2 \lambda^{(\varepsilon)} t \theta^{2}}\left(\mathbb{E}\left[n n^{\|}\right]-2 \lambda^{(\varepsilon)} \lambda^{\left.(\varepsilon) \|^{2}\right)}=\frac{\alpha \log 2}{2 \lambda^{(\varepsilon)} t \theta^{2}} \operatorname{Cov}\left(n, n^{\|}\right)=2 d \frac{\alpha \log 2}{\theta^{2}},\right.
\end{aligned}
$$

where we have used Lemma 4.5 .

$$
\begin{aligned}
m_{23}= & \frac{1}{2 \lambda^{(\varepsilon)} t} \mathbb{E}\left[\left(\frac{\partial l_{12}^{(\varepsilon)}}{\partial \alpha}\right)\left(\frac{\partial l^{(\varepsilon)}}{\partial \theta}\right)\right] \\
= & -\frac{1}{2 \lambda^{(\varepsilon)} t} \mathbb{E}\left[\left(\sum_{i=1}^{n}\left(\log \frac{Z_{i}}{\varepsilon}-\frac{1}{\alpha}\right)+\log \varepsilon\left(n-2 \lambda^{(\varepsilon)} t\right)\right)\left(\frac{\alpha \log 2}{\theta^{2}}\left(n^{\|}-\lambda^{(\varepsilon) \|_{t}} t+\sum_{i=1}^{n^{\|}}\left(T_{i}-\mu_{T}\right)\right)\right]\right. \\
= & -\frac{1}{2 \lambda^{(\varepsilon)} t} \mathbb{E}\left[\left(\sum_{i=1}^{n}\left(\log \frac{Z_{i}}{\varepsilon}-\frac{1}{\alpha}\right)\right)\left(\sum_{i=1}^{n^{\|}}\left(T_{i}-\mu_{T}\right)\right)\right] \\
& -\frac{\alpha \log 2}{2 \lambda^{(\varepsilon)} t \theta^{2}} \log \varepsilon \mathbb{E}\left[\left(n-2 \lambda^{(\varepsilon)} t\right)\left(n^{\|}-\lambda^{\left.(\varepsilon) \|_{t}\right)}\right]\right.
\end{aligned}
$$

Now note that the jumps $\left(X_{i}, Y_{i}\right)_{i=1, \ldots, n} \|$ are independent and independent of all single jumps in either component. Recall that $T_{i}=T\left(X_{i}, Y_{i}\right)=T\left(X_{i} / \varepsilon, Y_{i} / \varepsilon\right)$, where the last equality is easily checked. Hence, the right hand side above reduces to

$$
\begin{aligned}
& =-\frac{1}{2 \lambda^{(\varepsilon)} t} \mathbb{E}\left[\sum_{i=1}^{n^{\|}}\left(\log \frac{X_{i}}{\varepsilon}+\log \frac{Y_{i}}{\varepsilon}-\frac{2}{\alpha}\right)\left(T_{i}-\mu_{T}\right)\right]-\frac{\alpha \log 2}{2 \lambda^{(\varepsilon)} t \theta^{2}} \log \varepsilon \operatorname{Cov}\left(n, n^{\|}\right) \\
& =-\frac{\lambda^{(\varepsilon)} \|_{t}}{2 \lambda^{(\varepsilon)} t} \mathbb{E}\left[\left(\log \frac{X_{1}}{\varepsilon}+\log \frac{Y_{1}}{\varepsilon}-\frac{2}{\alpha}\right)\left(T_{i}-\mu_{T}\right)\right]-\frac{2 \lambda^{(\varepsilon)} \|_{t}}{2 \lambda^{(\varepsilon)} t} \frac{\alpha \log 2}{\theta^{2}} \log \varepsilon \\
& =-d\left(m+\frac{2 \alpha \log 2}{\theta^{2}} \log \varepsilon\right) .
\end{aligned}
$$

Finally, recalling $D=\left(d_{i j}\right)_{1 \leq i, j \leq 3}$,

$$
m_{33}=\frac{1}{2 \lambda^{(\varepsilon)} t} \mathbb{E}\left[\left(\frac{\partial l^{(\varepsilon)}}{\partial \theta}\right)^{2}\right]=-\frac{1}{2 \lambda^{(\varepsilon)} t} \mathbb{E}\left[\frac{\partial^{2} l^{(\varepsilon)}}{\partial \theta^{2}}\right]=d_{33} .
$$

